\title{
Hansische Geschichtsblätter
}

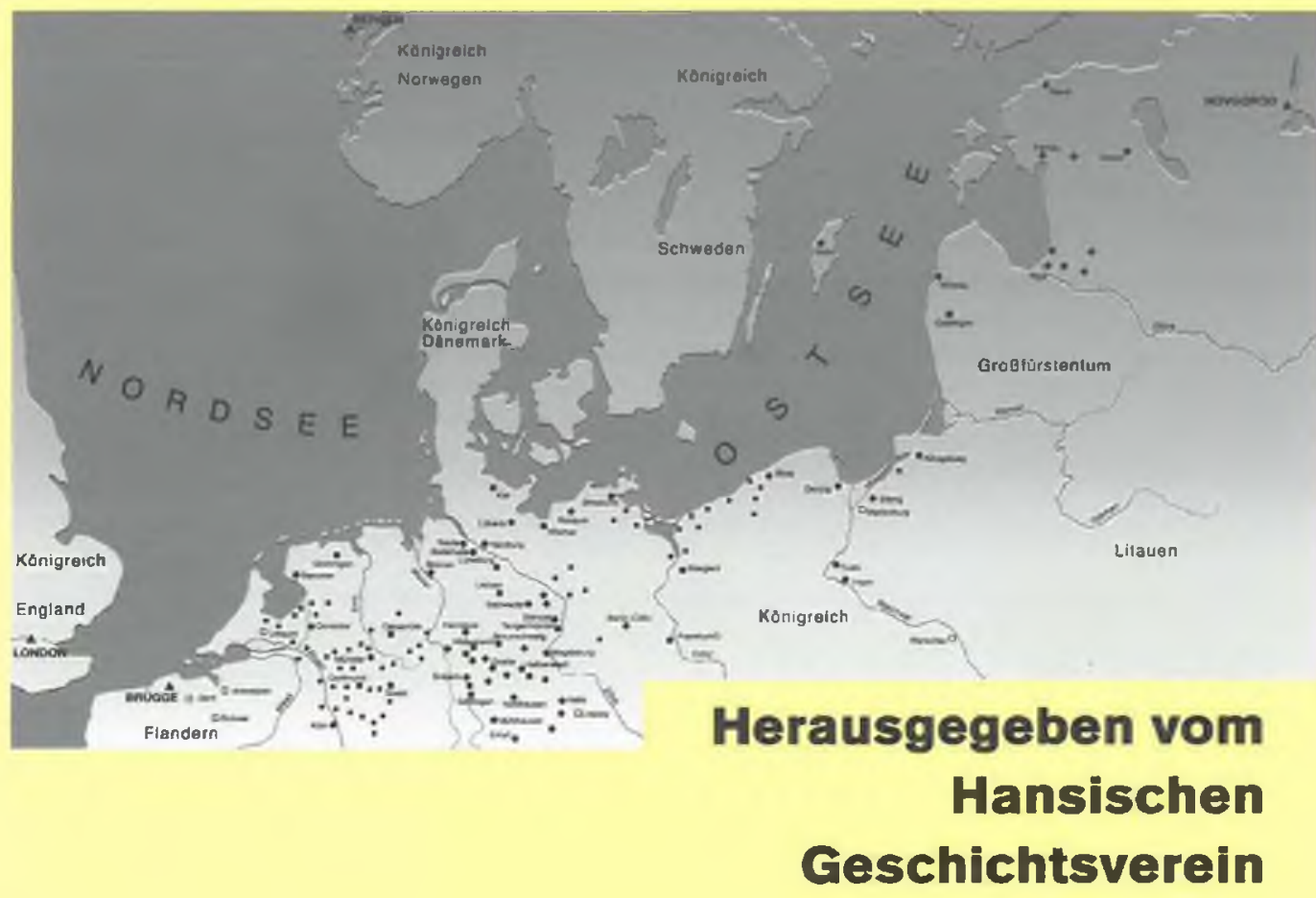

Porta Alba 


\title{
HANSISCHE \\ GESCHICHTSBLÄTTER
}

\author{
HERAUSGEGEBEN \\ VOM \\ HANSISCHEN GESCHICHTSVEREIN
}

131. JAHRGANG

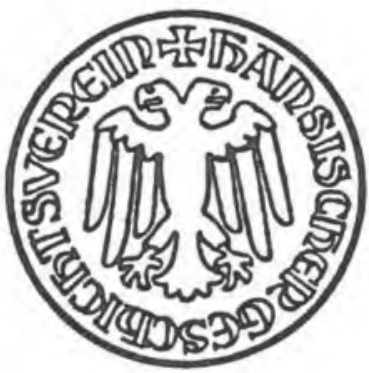

2013

Porta Alba Verlag

Trier 


\section{REDAKTION}

Aufsatzteil: Prof. Dr. Rolf Hammel-Kiesow, Lübeck

Umschau: Dr. Nils Jörn, Wismar

Für besondere Zuwendungen und erhöhte Jahresbeiträge, ohne die dieser Band nicht hätte erscheinen können. hat der Hansische Geschichtsverein folgenden Stiftungen. Verbänden und Städten zu danken:

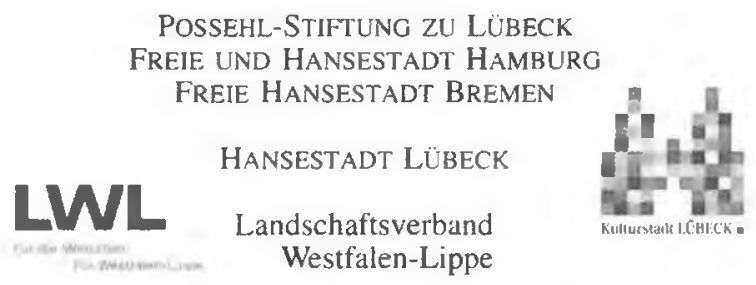

DR. MARGaReTE SCHINDLER, BuXTEHUdE

Die Hansischen Geschichtsblätter sind ein refereed journal. Eingereichte Beiträge unterliegen einem anonymisierten Begutachtungsverfahren (Double Blind Review), das über die Aufnahme in die Zeitschrift entscheidet.

Umschlagabbildung nach: Hanseraum und Sächsischer Städtebund im Spätmittelalter, in: Hanse, Städte, Bünde. Die sächsischen Städte zwischen Elbe und Weser, Bd. 1, hg. v. Matthias Puhle, Magdeburg 1996, S. 3.

Zuschriften, die den Aufsatzteil betreffen, sind zu richten an Herrn Prof. Dr. Rolf HAMMEL-KIESOW, Archiv der Hansestadt Lübeck, Mühlendamm 1-3, 23552 Lübeck (rolf.hammel-kiesow@luebeck.de); Besprechungsexemplare und sonstige Zuschriften wegen der Hansischen Umschau an Herm Dr. Nils Jörn, Archiv der Hansestadt Wismar, Altwismarstr. 7-17, 23966 Wismar (nilsjoern@aol.com)

http://www.hansischergeschichtsverein.de

Beiträge werden als Manuskript und auf Diskette erbeten. Die Verfasser erhalten von Aufsätzen und Miszellen 10, von Beiträgen zur Hansischen Umschau zwei Sonderdrucke unentgeltlich.

Die Lieferung der Hansischen Geschichtsblätter erfolgt auf Gefahr der Empfänger. Kostenlose Nachlieferung in Verlust geratener Sendungen erfolgt nicht.

Die in dieser Zeitschrift veröffentlichten Beiträge sind urheberrechtlich geschützt.

Eintritt in den Hansischen Geschichtsverein ist jederzeit möglich. Der Jahresbeitrag beläuft sich z. Zt. auf $€ 30$ (für in der Ausbildung Begriffene auf $€ 15$ ). Er berechtigt zum kostenlosen Bezug der Hansischen Geschichtsblätter. - Weitere Informationen gibt die Geschäftsstelle im Archiv der Hansestadt Lübeck, Mühlendamm 1-3, 23552 Lübeck.

ISSN 0073-0327

ISBN 978-3-93370l-49-7 


\title{
Die HANSE
}

\section{ÜBERLEGUNGEN ZUR ENTWICKLUNG DES HANSEBEGRIFFES} UND DER HANSE ALS INSTITUTION RESP. ORGANISATION

\author{
von Carsten Jahnke
}

Abstract: The Hanse: The development of the concept of the „Hanse“ and the development of the Hanse itself as an institution/organization.

How can we define the Hanse? When was it established? How did it develop institutionally and organizationally? A re-examination of the source material demonstrates that the Hanse did not exist until the mid-fourteenth century. To be sure, the term "German Hanse" makes its first appearance in 1358 (cf. Behrmann and Selzer), but that fact alone does not constitute compelling evidence that the Hanse already existed as an institution. As an institution, the Hanse saw the light of day first in a diet in Lübeck in June 1379. Prior to that, we can only talk about regional alliances and diets, which met ad hoc to deal with common economic matters. By contrast, from 1380 onwards general diets of the cities of the Hanse began to meet regularly, even if they also dealt with regional conflicts. Consequently, the term „Hanse“ must have developed organically between 1379 and 1418, starting as a loose term applicable to ad hoc gatherings, then being transformed into a name for an institution and then for an organisation. This fact requires us, however, to re-examine the role of the land-locked member towns of the Hanse. They were by no means grumbling subordinates to the coastal towns, for which older scholarship took them, but had a role of their own to play in the organization.

Die folgenden Thesen wurden im Anschluss an die 128. Pfingsttagung des Hansischen Geschichtsvereins in Lüneburg im Plenum heftig und intensiv diskutiert. Diese Skizzen mögen daher als Anregung zu weiteren Forschungen verstanden und keinesfalls als Endpunkt dieser Diskussion angesehen werden. 


\section{Der historiographische Hintergrund}

Die Entstehung der Hanse wird in der Forschung und vor allem in den Handbüchern zur hansischen Geschichte äußerst unterschiedlich behandelt. Dietrich Schäfer sah eine Entwicklung von der Hanse der deutschen Kaufleute zur Städtehanse, die sich 1356 vollzogen habe.' Vor ihm hatte Goswin von Brederlow das Entstehen der Hanse aus Sicht der preußischen Städte erst für das Jahr 1360 beschrieben. ${ }^{2}$ Arnold Kiesselbach dagegen sah die Hanse 1356 erst im Entstehen und zog eine klare Trennungslinie zwischen den Entwicklungen davor und danach. ${ }^{3}$ Mit den Forschungen Fritz Rörigs wurde die Entstehung einer wie auch immer gearteten Hanse oder einer Idee der Hanse in die Zeit um 1158 verlegt. ${ }^{4}$ Nach dem II. Weltkrieg datierte man die Entstehungsphase dann äußerst vage in die Mitte des 13. Jahrhunderts. ${ }^{5}$ Die neuere Forschung dagegen setzt den Beginn der „Deutschen Hanse“ in das Jahr 1358 und knüpft damit an die von Kiesselbach vorgezeichneten Linien an. "Dabei wird auch in vielen der neueren Werken konstatiert, dass es eine „Frühhanse" gegeben habe, die sich dann in die ,Deutsche Hanse" entwickelt habe.?

Diese Tendenz, der Hanse ein möglichst hohes Alter zu geben, ist teilweise dem Wissen geschuldet, dass die Institution resp. Organisation „Hanse“ eine lange Entwicklung aufweist; ${ }^{8}$ sie ist teilweise aber auch dem Umstand zu verdanken, dass unsere Hauptquellensammlung zu diesem Thema, die Hanserezesse, implizit den Beginn der Hanse auf das Jahr 1256 legen, wobei der Herausgeber Karl Koppmann die Entstehung der Hanse in ,der Verbindung deutscher Städte in der Heimath" sah. ${ }^{9}$ Bei der Auswahl seiner Quellen wählte Koppmann daher Texte, die ein „Erwachsen“ der Hanse zeigen sollten und er behandelte Anderes, wie z. B.

' Dietrich SCHÄFER, Die Blütezeit der Deutschen Hanse, 2 Bde, Berlin 1905, hier Bd. I, S. 13.

${ }^{2}$ Goswin von Brederlow, Geschichte des Handels der Ostsee-Reiche, Berlin 1820 , S. $43 \mathrm{f}$.

${ }^{3}$ Arnold KJesSElbaCH, Die wirtschaftlichen Grundlagen der deutschen Hanse und die Handelsstellung Hamburgs bis in die zweite Hälfte des 14. Jahrhunderts, Berlin 1907, hier S. 247-278.

${ }^{4}$ Fritz RÖRIG, Die Entstehung der Hanse und der Ostseeraum, in: DERS., Wirtschaftskräfte im Mittelalter, Weimar 1959, S. 542-603. Philippe Dollinger, Die Hanse, Stuttgart ${ }^{4} 1989$, S. 35-76.

${ }^{5}$ Klaus Friedland, Die Hanse. Stuttgart 1991, S. 125-130.

${ }^{6}$ Stephan SELzER, Die mittelalterliche Hanse, Darmstadt 2010, S. 50ff. Rolf HaMmELKIESOW, Die Hanse, München ${ }^{4} 2008$, S. $63 \mathrm{ff}$.

${ }^{7}$ Hammel-Kiesow, Die Hanse (wie Anm. 6), S. 38-59.

${ }^{8}$ Siehe zu diesen beiden Begriffen unten bei Anm. 15

${ }^{9}$ HR I, 1, S. XXV. 
"gegründete" Städtebünde, in diesem Zusammenhang nicht. ${ }^{10}$ Diese einseitige Auswahl unter einem sehr schlagkräftigen Titel, zusammen mit der anscheinend durchgängigen inneren Ordnung und Logik dieser Quellensammlung, hat das Bild der Hanse bis heute maßgeblich geprägt."

Im Folgenden soll versucht werden, im Anschluss an Stephan Selzer, Rolf Hammel-Kiesow und Arnold Kiesselbach, die Entstehung der Hanse noch einmal nachzuzeichnen. Es wird hierbei zum einen darum gehen, die bereits begonnene, kritische Auseinandersetzung mit den Hanserezessen als Quellenedition fortzuführen. Zum anderen soll versucht werden, die Entwicklungsprozesse, die zum Entstehen der Hanse geführt haben, nachzuzeichnen.

Dabei wird als Hauptthese konstatiert werden, dass die Hanse sich als feste Institution im Zeitraum zwischen 1379 und 1380 etablierte und erst im 15. Jahrhundert zu einer Organisation wurde. Es wird gezeigt, dass wir vorher nicht von „der Hanse“, „der Hanse der Kaufleute“ oder anderen Protoinstitutionen sprechen können.

Die hier vorgestellten Ausführungen sind, wie auch die Hanse, natürlich nicht aus einem Vakuum entstanden. Sie basieren, auch wenn dieses nicht immer explizit vermerkt wurde, auf den Gedanken und Anregungen vieler einzelner Kollegen, denen ich zu großem Dank verpflichtet bin, oder, wie Bernhard von Clairvaux es ausdrückte, wir sind alle Zwerge auf den Schultern von Giganten: Dicebat Bernardus Carnotensis, nos esse quasi nanos gigantium humeris insidentes. ${ }^{12}$ Es soll daher ausdrücklich darauf hingewiesen werden, dass es nur die kollegiale Zusammenarbeit innerhalb des Hansischen Geschichtsvereines war, die diese Thesen überhaupt erst möglich machte.

\section{Methodische Vorüberlegungen}

Die folgenden Ausführungen werden versuchen, auf zwei Wegen das Entstehen der Hanse nachzuzeichnen. Zum einen sollen die in den Hanserezessen von Koppmann veröffentlichten Quellen noch einmal einer inhaltlichen Analyse unterzogen werden. Hierbei wird es vor allem darauf ankommen, welche persona agens oder institutiones agentes genannt werden, wie sie sich selbst benennen und wie ihr Verhältnis zu anderen Gruppen

${ }^{10} \mathrm{Zu}$ den Problemen und Gefahren der Hanserezesse siehe Angela HuANG und Ulla KYPTA. Ein neues Haus auf altem Fundament. Neue Trends in der Hanseforschung und die Nutzbarkeit der Rezessedition, in: HGbll. 129, 2011, S. 213-230, hier S. 221-225.

"Angela HUANG und Ulla KYPTA, Ein neues Haus auf altem Fundament (wie Anm. 10).

${ }^{12}$ Johannes von Salisbury, Metalogicon III, 4, fol. 900a, in: loannis Saresberiensis episcopi Carnotensis Metalogicon, Libri III, hg. v. Clemens C. I. Webb, Oxford 1929, S. 136, $23 \mathrm{ff}$. 
aussieht. Diese Quellen sollen mit anderen Texten ergänzt werden, die von Karl Koppmann nicht in die Ausgabe der Hanserczesse aufgenommen wurden. Diese Vorgehensweise unterscheidet sich dabei von anderen Beiträgen zum Wesen der Hanse, die, zumeist vom 16. Jahrhundert ausgehend, den Hansebegriff zurückverfolgen und damit spätere Entwicklungen in die Frühzeit projizieren. ${ }^{13}$

Zum zweiten wird gefragt werden, welche spezifischen Aufgaben und Funktionen die einzelnen Gruppen und Organisationen übernommen haben, die sich in den Quellen finden lassen und, ob ihnen ein institutioneller Charakter zugeordnet werden kann. ${ }^{14}$

Es werden im Vorwege keine Theorien aufgestellt, was die Hanse gewesen sei. Es soll vielmehr gefragt werden, was die Quellen selbst sagen, mit allen Widersprüchlichkeiten und Unklarheiten.

Die folgenden Überlegungen basieren aber auf den von Douglass C. North entwickelten Theorien zu Institutionen resp. Organisationen. Sehr vereinfacht gesagt wird im Folgenden unter Institution eine Einrichtung verstanden werden, die die ökonomischen und politischen Interaktionen zwischen Kaufleuten resp. deren Städten reguliert, um dadurch Transaktionskosten zu minimieren und durch koordiniertes und kooperatives Verhalten Gewinne zu maximieren. Im ökonomischen Bereich sind Institutionen vor allem dort von besonderer Bedeutung, wo familiäre und soziale Bindungen gelockert oder nicht vorhanden sind. ${ }^{15}$ Sehr verkürzt sichern Institutionen im Innern den Handel untereinander, normieren und regulieren ihn, um damit Kosten zu sparen, und sichern nach Außen den Handel durch Abschluss von Verträgen, die die Such-, Meß- und Gerichtskosten reduzieren.

An dieser Stelle soll aber noch einen Schritt weiter gegangen werden, in dem zwischen Institutionen im Sinne Norths und Organisationen unterschieden werden soll. Hierbei wird unter einer Institution eine lose und nicht konstitutionalisierte Einrichtung verstanden. Eine Organisation da-

${ }^{13}$ Siehe u. a. Eva-Marie DISTLER, Städtebünde im deutschen Spätmittelalter. Eine rechıshistorische Untersuchung zu Begriff, Verfassung und Funktion (Studien zur europäischen Rechtsgeschichte, Bd. 207), Frankfurt am Main 2006, S. 53-68. Siehe auch Thomas BEHRMANN, ,Hansekaufmann', .Hansestadt", .Deutsche Hanse“. Über hansische Terminologie und hansisches Selbstverständnis im späten Mittelalter, in: Bene vivere in communitate. Beiträge zum italienischen und deutschen Mittelalter. Hagen Keller zum 60. Geburtstag, hg. v. Thomas Scharff und Thomas Behrmann, Münster 1997, S. 155-176.

is Siehe hierzu Stuart JENKS, Transaktionskostentheorie und die mittelalterliche Hanse, in: HGbll. 123, 2005, S. 31-42. Douglass C. NORTH, Transaction Costs in History, in: Journal of European Economic History, Vol. XIV, 1985, S. 557-576.

${ }^{15}$ Douglass C. NORTH, Institutions, in: Journal of Economic Perspectives, Vol. 5, Nr. 1, Winter 1991, S. 97-112, hier besonders S. 97-102 sowie 105ff. S. a. DERS., Institutions, institutional change and economic performance, Cambridge 1990, S. 4 ff. 
gegen soll eine Einrichtung bezeichnen, die durch konstitutionelle, auch zeremoniell-konstitutionelle Vorgänge, Satzungen oder Verhaltensnormen so weit verfestigt ist, dass sie eine eigene, beständige Existenz aufweist. ${ }^{16}$ Institutionen in diesem Sinn werden ad hoc von den Mitgliedern heraus gebildet, Organisationen sind stetig und versuchen, auf ihre Mitglieder konstant einzuwirken.

\section{Die Zeit zwischen 1150 und 1252: Die Kaufleute}

Wie neuere Forschungen gezeigt haben, schlüpfte die Stadt Lübeck nicht fïx und fertig aus dem Ei, parat, ihre führende Rolle im Ostseeraum sofort und mit Macht zu übernehmen. ${ }^{17}$ Insofern ist Rörigs Theorie einer „,metaphysischen" Hanse, die sich 1158 mit dem Entstehen des modernen Lübecks manifestiert habe, heute widerlegt. Auch scheint es sicher zu sein, dass wir nicht mehr von der „Gotländischen Genossenschaft" und einer Sonderrolle der Deutschen auf Gotland im 12. Jahrhundert sprechen können. ${ }^{18}$

Das, was man für das 12. Jahrhundert konstatieren kann, ist, dass sich ins Ausland reisende Kaufleute in wehrhaften Gruppen, dem ,félag" oder der ,hansa", zusammengeschlossen haben, so wie sie es schon seit dem ausgehenden 8. Jahrhundert getan hatten. Diese Guppen hatten im Ausland Privilegien erworben, die landsmännisch oder nach Städten organisiert waren. So gab es z. B. die Privilegien der Dänen oder der Kölner in England. ${ }^{19}$ Zudem hatte z. B. der dänische König erfolgreich versucht, die verschiedenen Gruppen seiner ins Ausland reisenden Kaufleute in einer Gilde, der St. Knuds-Gilde, zu vereinen. ${ }^{20}$ Dieses verlieh den Kaufleuten

\footnotetext{
${ }^{16}$ NorTH, Institutions (1990) (wie Anm. 15), S. 4 ff.

${ }^{17}$ Siehe zuletzt Carsten JAHNKE. Handelsstrukturen im Ostseeraum im 12. und beginnenden 13. Jahrhundert. Ansätze einer Neubewertung, in: HGbll. 126, 2008, S. 135-175.

${ }^{18}$ Carsten JAHNKE, „Homines imperii“" und „osterlinge“. Selbst- und Fremdbezeichnungen hansischer Kaufleute im Ausland am Beispiel Englands, Flanderns und des Ostseeraumes im 12. und 13. Jahrhundert, in: HGbll. 129, 2011, S. 1-57.

${ }^{19}$ JAHNKE, Homines imperii (wie Anm. 18), S. 8-13.

${ }^{20}$ Camillus NyroP, Danmarks Gilde- og Lavskraaer fra Middelalderen, Kjöbenhavn 1899-1900, Bd. I, Nr. 1-2, S. 1-6. Im Gegensatz zu Kurt Villads JenSEN, Denmark and the Second Crusade: the formation of a crusader state? In: Jonathan Phillips u. Martin Hoch (Hrsg.), The Second Crusade. Scope and consequences. Manchester \& New York 2001, S. 164-179, soll an dieser Stelle an der Interpretation der Knuds-Gilden als Kaufmannsgilden festgehalten werden. Weder die uiberlieferten Gildeschraen noch die Verlegung der Generalsynode der Gilde von Ringsted auf die Schonischen Messen im Jahre 1256 sprechen für eine direkte Verbindung der Gilde mit Kreuzfahrern. Siehe hierzu ausführlich Lauritz WEIBULL, S:t Knut i Österled, in: Scandia, Bd. XVII, 1946, S. 84-101. Hans Torben Gilkier, In honore Sancti Kanuti martyris. Konge og Knudsgilder i det 12. århundrede, in: Scandia, Bd. XLVI, 1980, S. 121-162, u. Thomas RiIS, Die dänischen Knudsgilden als Handelsorganisation, in: Regna firmat pietas. Staat und Staatlichkeit im Ostseeraum. Festgabe zum 60. Geburtstag von Jens E. Olesen, hrsg. v. Martin Krieger u. Joachim Krüger, Greifswald 2010. S. 33-45.
} 
den besonderen Schutz des Königs und seines Namens und verhalf der Königsmacht zu Ehre, Einkünften durch Abgaben und zur Erhaltung ihrer Macht. Eine vergleichbare Gilde ist aus dem Bereich der späteren Hanse nicht bekannt.

Was wir dagegen feststellen können ist, dass sich die aus dem nördlichen Heiligen Römischen Reich kommenden Kaufleute im Ausland seit dem Beginn des 13. Jahrhunderts zu größeren Gruppen zusammenschlossen, um so ihre Marktposition zu sichern. Die Entwicklung kann u. a. für London und Visby sichtbar gemacht werden. ${ }^{2 !}$ Diese Erkenntnis ist durchaus nicht neu. Der Zusammenschluss erfolgte freiwillig und unabhängig von den Ausgangsstädten, d. h., die Institutionen der Kaufleute waren Erscheinungen vor Ort, die keine direkte Beziehung zu den Ausgangsstädten besaßen. Im Sinne der Institutionenökonomie ${ }^{22}$ ersparten sie den Kaufleuten Transaktionskosten, ${ }^{23}$ Aufwendungen für die Marktsuche, die Rechtssicherheit, den Marktfrieden etc. und garantierten gleichzeitig dem Herrscher geregelte Einkünfte.

Eine Zwitterstellung zwischen Kaufleuten und Städten nahmen die „deutschen“ Kaufleute in Brügge ein. Hier war es vor allem die Stadt Lübeck, die 1252 die Initiative übernommen hatte, für ihre und die Kaufleute anderer Städte Privilegien zu erwerben. ${ }^{24}$ Dieses sollte später als ein Katalysator für die Bildung der Hanse dienen, ohne dass dieses von vorne herein intendiert war.

Die Institutionen der Kaufleute im Ausland erhielten Namen, die der entsprechenden Tradition vor Ort und der besonderen Entwicklung an jedem einzelnen Ort entsprachen. In England entwickelte sich um $1280 \mathrm{der}$ Begriff der „mercatores de hansa Alman[ie]“ der 1282 mit dem Zusatz, dass dies die Kaufleute aus verschiedenen Teilen Deutschlands seien, die in dieser Stadt London zusammenkämen, mercatores ... de partibus Alman/ie] ad eadem civitatem [i.e. London] confluentes, erklärt wurde. ${ }^{25}$ Der Begriff der Kaufleute der deutschen Hanse war in England um 1280 also neu und bis dahin ungehört. In Flandern erschufen die Lübecker 1252 den Begriff der Vereinigten Kaufleute des Römischen Reiches, „universi mercatores Romani imperii“", ${ }^{26}$ ebenso eine Neuschöpfung wie in London.

\footnotetext{
${ }^{21}$ JAHNKE, Homines imperii (wie Anm. 18).

${ }^{22}$ Siehe hierzu grundlegend NORTH, Institutions (1990) (wie Anm. 15), S. 4 ff. Siehe auch DERS., Institutions (wie Anm. 15), S. 97-112.

${ }^{23}$ Siehe hierzu JENKS, Transaktionskostentheorie (wie Anm. 14), S. 36-42.

${ }^{24}$ JAHNKE, Homines imperii (wie Anm. 18), S. 24-29.

${ }^{25}$ HUB I, Nr. 902, S. 308 ff., hier S. 309. Siehe JAHNKE. Homines imperii (wie Anm. 18), S. 23.

${ }^{26}$ HUB I, Nr. 428, S. 140 f. Walther STEIN, Über die ältesten Privilegien der deutschen Hanse in Flandern und die ältere Handelspolitik Lübecks, in: HGbll, XXX, 1903. S. 49-133. hier S. 78.
} 
In Visby kennen wir den Namen der Institution nicht. In den Urkunden bezeichnet sie sich als Gruppe aller Kaufleute aus verschiedenen Städten und Orten, die das Land Gotland besuchen, als Gruppe der ,omnium mercatorum diversarum civitatum et locorum terram Gotlandiam frequentacium “. ${ }^{27}$ Diese Gruppe soll der Einfachheit halber als Kaufleute im Staven von Visby bezeichnet werden. ${ }^{28}$

Ökonomisch gesehen hatten die Kaufleute an allen Orten im Ausland mit den gleichen oder ähnlichen Problemen der Transaktionskostenbewältigung zu kämpfen und sie kamen in der Zeit zwischen 1150 und 1250 auch an allen Orten zu gleichen oder ähnlichen Lösungen. Dieses galt nicht nur für die Kaufleute aus dem Heiligen Römischen Reich, sondern für alle fremden Kaufleute im Ausland. Auch kann man davon ausgehen, dass sich die Entwicklungen an den einzelnen Orten gegenseitig beeinflussten und, dass die Kaufleute einzelner Städte durchaus an verschiedenen Orten handeln konnten, wie das Beispiel aus dem Jahr 1351 zeigt, als einige Kaufleute in England beschwören, dass sie dieses eben nicht täten. ${ }^{29}$ Diese Erkenntnis ist altbekannt. Allerdings haben wir keinen Beleg über eine übergreifende Institution, die ordnend im Hintergrund eingegriffen hätte, weder von Seiten der Kaufleute noch von Seiten der Städte. Zudem galten die institutionellen Vorteile nur vor Ort, d. h. ein Kaufmann, der Mitglied der jeweiligen Hanse in England war, kam damit nur in den Genuss der Vorteile in England. Die Institution hatte keine Auswirkungen und keinen Einfluss z. B. auf die Produktion von Waren auf dem Kontinent, ebensowenig wie anderen Ortes über die Aufnahme von Kaufleuten am Londoner Kontor entschieden wurde. Wir können daher nur von regionalen Institutionen sprechen, die noch nicht das Stadium einer regionenübergreifenden Organisation erreicht hatten. ${ }^{30}$

\section{Die Zeit zwischen 1150 und 1250: Die Städte}

Karl Koppmann ging bei seiner Zusammenstellung der Texte für die Hanserezesse von den Städten als treibende Kräfte für die Entstehung der Hanse aus und spricht den Kaufleuten keine Rolle zu. ${ }^{31}$ Es stellt sich also die Frage, welche Funktion den Städten bei der Entwicklung zukam.

\footnotetext{
${ }^{27}$ HUB I, Nr. 1024, S. 354f.

${ }^{28}$ Siehe JaHNKE, Homines imperii (wie Anm. 18), S. 29-47.

${ }^{29}$ HR I, 1, Nr. 148, S. $83 \mathrm{f}$.

${ }^{30}$ Siehe zum Unterschied von Institution und Organisation NORTH, Institutions (1990) (wie Anm. 15), S. 4f.

${ }^{31}$ Siehe z. B. HR I, I, S. 74, Kommentar zu Nr. 143.
} 
Hierbei gab es mehrere Entwicklungen parallel zueinander. Erstens führte die nachlassende Kraft des Kaisertums, eine innere Ordnung im Reich zu garantieren, dazu, dass sich u. a. die Städte überall im Heiligen Römischen Reich zu Verteidigungsbünden zusammenschlossen, die immer größere Räume umfassten. Beispiele hierfür sind die Annäherungen zwischen Hamburg und Lübeck in den 1230er Jahren ${ }^{32}$ sowie der Rheinische Städtebund von 1254-1256, der bis nach Hamburg und Lübeck reichte. ${ }^{33}$ Viele dieser Bündnisse waren von kurzer Dauer und abhängig von der aktuellen politischen Situation. Sie erhöhten die Kontaktdichte zwischen den Städten und sie bewiesen, dass gemeinsames Handeln erfolgreich sein konnte. Gleichzeitig hatten die Städtebünde verschiedene Zielrichtungen: sie verbanden Reichs- und Sicherungspolitik mit den handelspolitischen Interessen der beteiligten Städte. ${ }^{34}$ Allerdings lässt sich bis 1250 kein durchgehendes, einheitliches Interesse einer Städtegruppe nachweisen, wie z. B. die Angriffe und teilweise Zerstörung Stralsunds und Kopenhagens durch Lübeck 1249 deutlich zeigen.

Zum zweiten wuchsen einige Städte durch Rechtsgleichheit zu relativ festen Gruppen zusammen. Ein Beispiel hierfür ist Lübeck, welches als Oberhof der Städte des lübischen Rechts im 13. Jahrhundert einen engen Kontakt mit ",seinen“ mindestens 35 Städten aufrecht erhielt. ${ }^{35}$ Die Rechtsgleichheit implizierte dabei auch ökonomische Aspekte, da sie im institutionellen Sinn die Rechts- und Handelskosten minimierte. ${ }^{36}$ Gleichzeitig ließen sich Rechtskonsultationen und -tage auch zur politischen Einflussnahme nutzen.

Diese Entwicklungen bilden den Hintergrund der nun folgenden Ereignisse. In sich selbst zeigen sie aber keine Anzeichen dafür, dass sich in

\footnotetext{
32 JAHNKE, Handelsstrukturen (wie Anm. 17), S. $167 \mathrm{ff}$.

${ }^{33}$ Christian MülLER, Lübeck und der Rheinische Städtebund 1254-1256. Formen und Möglichkeiten städtischer Politik an der Wende zum Spätmittelalter, in: ZVLGA 80, 2000, S. 165-184. Siehe auch Siegfried EPPERLEIN, Städtcbünde und Feudalgewalten im 13. Jahrhundert. Die Beziehungen der in Bünden und Landfrieden vereinigten Städte zu fürstlichen Gewalten und zum deutschen Königtum, in: ZfG 20, 1972, S. 695-718, hier S. 698-702. Siehe auch Jürgen Karl W. BERNS, Propter communem utilitatem, Studien zur Bündnispolitik der westfalischen Städte im Spätmittelalter (Studia Humaniora, Bd. 16), Düsseldorf 1991, S. 45 .

${ }^{34}$ MüLlER, Lübeck und der Rheinische Städtebund (wie Anm. 33), S. 170 und $181 \mathrm{ff}$. Siehe auch Evamaria ENGEL, Städtebünde im Reich 1226-1314 - eine vergleichende Betrachtung, in: Bürgertum - Handelskapital - Städtebünde, hg. v. Konrad Fritze. Eckhard Müller-Mertens und Johannes Schildhauer (Hansische Studien, Vol. III, Abhandlungen zur Handels- und Sozialgeschichte, Bd. 15), Weimar 1975, S. 177-209, hier S. 178 und 180.

${ }^{35}$ Carsten JAHNKE, Der Aufstieg Lübecks und die Neuordnung des südlichen Ostseeraumes im 13. Jahrhundert, in: Städtelandschaften im Ostseeraum im Mittelalter und in der Frühen Neuzeit, hg. v. Roman Czaja und Carsten Jahnke (Towarzystwo Naukowe w Toruniu, Wyd. 1. Ark. wyd. 12), Torun 2009, S. 29-72, hier S. 58-69.

${ }^{36}$ JAHNKE, Aufstieg Lübecks (wie Anm. 35), S. $37 \mathrm{ff}$.
} 
ihnen die Hanse präkonstituieren würde. Ganz im Gegenteil. Die rheinischen, westfälischen, preußischen oder wendischen Städte zeigten weder eine innere Geschlossenheit, noch ein langanhaltendes Interesse an gemeinsamen politischen oder wirtschaftlichen Fragen. Hierzu bedurfte es weiterer Entwicklungen.

\section{Die Entwicklungen zwischen 1250 und ca. 1350}

Schaut man sich jetzt die Urkunden und Begebenheiten an, die Karl Koppmann in den Hanserezessen gesammelt hat, so werden verschiedene, ebenfalls parallele Entwicklungen deutlich.

Als erstes sind ein Großteil der abgedruckten Urkunden Dokumente verschiedener Städtegruppen oder -bünde, die Koppmann in seiner Auswahl vor allem im Küstenbereich der Ostsee gesehen hat, wie eine Übersicht der ersten zehn Stücke der Hanserezesse zeigt.

Es wird am Beispiel Lübecks - und das ist nur ein Beispiel - deutlich, dass sich das Rechtsgebiet des lübischen Rechts und die politischen und handelspolitischen Interessenfelder in diesem Raum zu decken begannen. Lübeck nutzte seine Position als Oberhof, um auch handelspolitische Fragen zu besprechen. So stellt der Wismarer Rechtstag von 1260 einleitend fest, dass man an dem festzuhalten wünsche, was man zum Wohle der Kaufleute, die sich des lübischen Rechtes erfreuen und diesem unterliegen, beschliessen werde. ${ }^{37}$ Das Bündnis galt also für diejenigen Kaufleute, die dem lübischen Recht unterstanden, wodurch die besondere Kopplung zwischen diesem und der Kaufmannschaft mehr als deutlich wird. Ein ähnliches Argumentationsmuster finden wir 1297 wieder, als sich die Städte lübischen Rechts gegen Probleme wehren, die den gemeinen Kaufleuten, den „communes mercatores“, in Flandern gemacht wurden. ${ }^{38}$ Die Städte lübischen Rechts fassen sich also als eine besondere Einheit auBerhalb ihrer territorialen Verbindungen auf.

Sie selbst bezeichnen sich dabei seit ca. 1284 als die Seestädte, vereinigt in einem Bündnis zur Wahrung des Friedens, civitates maritimae, comprehenses in confederacione concepte pacis, ${ }^{39}$ oder kurz als civitates maritimae, als Seestädte. ${ }^{40}$ Innerhalb dieser Gruppe kristallisiert sich in der zweiten Hälfte des 13. Jahrhunderts eine Führungsgruppe heraus, die

\footnotetext{
${ }^{37}$ MUB II, Nr. 873, hier S. $156=$ HR I, 1, Nr. 7, S. 4. ad audienciam uestram peruenire cupimus de arbitrio, quod discreto proborum uirorum cum deliberacione prouida statuit in subsidium omnium mercatorum, qui iure Lubicensi gaudent et reguntur

${ }^{38}$ HR I, 1, Nr. 77, S. 38 .

${ }^{39}$ HR I, 1, Nr. 30. S. 18f. Siehe auch Liv-, Esth- und Kurländisches UB. 1.2, Nr. DCXIII, Sp. 19.

${ }^{40}$ HR I, 1, Nr. 34, S. 20f. und Nr. 80, S. 41 f.
} 
Abb. I. Übersicht über die ersten elf Stücke der Hanserezesse

\begin{tabular}{|l|l|l|}
\hline \multicolumn{1}{|c|}{ Datum } & \multicolumn{1}{|c|}{ Art } & \multicolumn{1}{|c|}{ Nummer in den HR } \\
\hline 1256 & $\begin{array}{l}\text { Schadensersatz zwischen } \\
\text { Lübeck und Rostock als } \\
\text { Resultat des Krieges von } \\
1253\end{array}$ & \\
\hline 1259 & $\begin{array}{l}\text { Städtebündnisse Lübeck, } \\
\text { Rostock, Wismar, Wolgast } \\
\text { sowie Lübeck, Hamburg }\end{array}$ & $3-5$ \\
\hline 1260 & $\begin{array}{l}\text { Rechtstag und Bekämp- } \\
\text { fung von Piraten, Wismar, } \\
\text { Lübeck, Stral- } \\
\text { sund, Demmin }\end{array}$ & $7-8$ \\
\hline 1265 & $\begin{array}{l}\text { Rechtstag und Bekämp- } \\
\text { fung von Räubern. } \\
\text { Vereinbarung } \\
\text { regelmäßiger Treffen }\end{array}$ & $\begin{array}{l}9 \text { (Die von Koppmann in } \\
\text { Nr. 10 konstruierte Ver- } \\
\text { sammlung von 1277 hat } \\
\text { nicht stattgefunden. HR } \\
10 \text { ist ein Beschluss aus } \\
\text { dem Staven von Visby.) }\end{array}$ \\
\hline 1281 & $\begin{array}{l}\text { Schlichtung von Streitig- } \\
\text { keiten zwischen Greifs- } \\
\text { wald und Stralsund durch } \\
\text { Lübeck, Wismar und Ros- } \\
\text { tock }\end{array}$ & \begin{tabular}{l}
11 \\
\hline
\end{tabular} \\
\hline
\end{tabular}

von Lübeck, Hamburg, Rostock, Wismar, Stralsund und Greifswald gebildet wird. Allerdings beruht das Bild einer Sonderrolle der „civitates maritimae" allein auf der Auswahl Koppmanns, der sich vor allem auf die Seestädte konzentriert hat.

Schaut man zum Beispiel nach Westfalen, so bildet sich auch dort im 13. Jahrhundert eine Gruppe aktiver Städte heraus, die mehr und mehr zusammenarbeiten und die u. a. durch Münster, Dortmund, Soest und Lippstadt sowie Osnabrück und Minden gebildet wird. ${ }^{41}$ Die Struktur und

${ }^{41}$ Dortmunder UB, 1.1, Nr. 96, S. 40f., Nr. 104. S. 47, Nr. 106, S. 48f., Nr. 116. S. 53f., Nr. 127, S. 62 f., Nr. 133, S. 66 f., Nr. 134, S. $67 f$ f, Nr. 146, S. 81 f., Nr. 152, S. 84 f. etc. Siehe auch Urkundenbuch der Stadt Osnabrück 1301-1400, bearbeitet von Horst-Rüdiger Jarck. Osnabrück 1989, Nr. 44, S. 50. Siehe weiterhin Heinrich SCHOPPMEYER, Hansische Organisationsformen in Westfalen, Entwicklung und Struktur, HGbll. 100, 1982, S. 69-86, der den ersten Städtebund zur Sicherung von Kaufmmansinteressen schon in das Jahr 1246 legen kann, hier S. 71. Weiterhin umfassend BERNS, Propter communem utilitatem (wie Anm. 33), u. a. S. 24-32. Siehe generell Volker HENN. Städtebünde und regionale Identitäten im han- 
das Agieren der Städte untereinander und nach außen entsprechen dabei der der sogenannten wendischen Städte. ${ }^{42}$ Gleiche Bündnisstrukturen lassen sich auch für andere Gebiete des späteren Hanseraumes, z. B. für die der sächsischen, rheinischen oder preußischen Städte, finden. So setzt in Sachsen die Bündnispolitik schon in der Mitte des 13. Jahrhunderts ein, und der Bund der sächsischen Städte umfasste dabei u. a. Goslar, Lüneburg. Braunschweig, Hildesheim, Einbeck, aber auch Magdeburg, Stendal, Tangermünde, Werben, Salzwedel und Seehausen i. A. ${ }^{43}$ Es ist also irrig zu meinen, wie es die Hanserezesse suggerieren, dass allein das Bündnis der wendischen Städte die Hanse hervorgebracht habe.

Weiterhin begannen die Städtegruppen regulierend auf die Produktion und die Produktionskosten einzuwirken und damit als Institutionen zu wirken, indem sie Ordnung garantierten und Unsicherheit im Austausch zu beseitigen versuchten. ${ }^{44}$ Ein bekanntes Beispiel hierfür ist die 1321 von den wendischen Städten gemeinsam beschlossene und dann in den einzelnen Städten eingeführte Böttcherrolle ${ }^{45}$ oder aber die $1335 / 36$ von den preußischen Städten geschlossenen Vereinbarungen über einheitliche Maße und Gewichte. ${ }^{46}$ Es wird deutlich, dass zu einem Zeitpunkt um 1300 die Städtebünde eine weitere institutionelle Funktion übernahmen: Vereinheitlichung der Produktionsnormen und -preise und verbunden damit die Senkung der Ausgaben für Messkosten. In diesem Bereich wären noch weitergehende Forschungen notwendig, um den Zeitraum zu ermitteln, in dem diese Art der Zusammenarbeit begonnen hat.

Darüber hinaus gab es innerhalb und zwischen den Städtegruppen Bestrebungen, den Handel zwischen und unter ihnen zu erleichtern. So beenden z. B. Hamburg und Köln 1258 ihre alte Zwietracht, und Köln gelobt, die Hamburger Kaufleute zu fördern, ${ }^{47}$ Hamburg fördert die bremischen Kaufleute an der Elbe, ${ }^{48} 1275$ schließen Stade und Bremen einen

sischen Raum, in: Regionale Identität und soziale Gruppen im deutschen Mittelalter, hg. v. Peter Moraw (ZHF, Beiheft 14), Berlin 1992, S. 41-64, hier S. 44-56.

${ }^{42}$ Siehe die inhaltlichen Ausführungen bei SCHOPPMEYER. Organisationsformen in Westfalen (wie Anm. 41), S. 72ff. und 75f. Siehe auch ENGEl, Städtebünde (wie Anm. 34), S. 180f.; EPPERLEIN, Städtebünde (wie Anm. 33), S. 709-714.

${ }^{43}$ Siehe ausführlich hierzu Matthias PuHLE, Der sächsische Städtebund. Entstehung und Wirkung, in: Hanse, Städte, Bünde. Die sächsischen Städte zwischen Elbe und Weser um 1500, hg. v. Dems., Magdeburg 1996, Band 1, S. 15-28; siehe auch HENN, Städtebünde (wie Anm. 41), S. $57 \mathrm{f}$.

North. Institutions (1991) (wie Anm. 15), S. 97.

${ }^{45}$ HR I, 1, Nr. 105ff., S. 57-60.

${ }^{46}$ Acten der Ständetage Preußens, Vol. I, Nr. 14, S. 32 ff.

${ }^{47}$ HUB I, Nr. 513, S. 179.

${ }^{48}$ HUB I, Nr. 517, S. $180 \mathrm{f}$. 
Vertrag, ${ }^{49} 1298$ Hildesheim und Hannover, ${ }^{50} 1301$ Bremen und Hannover, ${ }^{51}$ und die Utrechter fördern die Kölner in ihrer Stadt. ${ }^{52}$ Diese Bestrebungen umfassten nicht alle Städte gleichzeitig und umfassend. ${ }^{53}$ So klagte Dortmund zum Beispiel noch bis 1332 darüber, in Hamburg und Lübeck entgegen aller Privilegien mit Zöllen und Abgaben belastet zu werden. ${ }^{54}$

Ebenso gab es ein reges Interesse in vielen Städten des nördlichen Heiligen Römischen Reiches am Handel mit dem Ausland. Zum einen, da viele begannen, die ausländischen Märkte als Absatzorte ihrer eigenen Produkte aufzufassen, ${ }^{55}$ und zum anderen, da ihre Kaufleute aktiv und direkt ausländische Märkte besuchten. So werden 1295 bei den Diskussionen um die Verlegung des Oberhofes für den Novgoroder St. Peterhof dreißig Städte mit dem Handel dort direkt in Verbindung gebracht: Dortmund, Köln, Magdeburg, Halle, Braunschweig, Wismar, Rostock, Goslar, Danzig, Stade, Greifswald, Kiel, Elbing, Lippstadt, Paderborn, Lemgo, Herford, Minden, Stralsund, Höxter, Hildesheim. Hannover, Lüneburg, Reval, Osnabrïck, Visby, Riga, Soest, Münster und Lübeck. Und hierbei handelt es sich nur um eine Auswahl der wichtigsten Städte. ${ }^{56}$ Bis weit ins 14. Jahrhundert hinein finden wir viele Kaufleute auch kleinerer Städte, vor allem auch des Binnenlandes, direkt auf den ausländischen Märkten. Dieses führte auf Dauer zu einer Zusammenarbeit zwischen den verschiedenen Regionen.

Vor diesem Hintergrund wirkten drei weitere Entwicklungen als anregende Kräfte, die die verschiedenen Städtegruppen zu einer einheitlichen Institution zusammenführen sollten.

Als erstes führten die Ereignisse in Flandern dazu, dass die Einigungstendenzen der verschiedenen Städtegruppen und -bünde verstärkt wurden. Als die Lübecker 1252 die flandrischen Privilegien verhandelten, zwangen sie durch den Abschluss indirekt alle beteiligten Kräfte in Zukunft zu einer gemeinsamen Vorgehensweise. Die Lübecker hatten. wahrscheinlich, um nicht durch die großen und alten Handelsstädte im Raum unterdrückt zu werden, als Privilegienempfänger die Gemeinschaft der Kaufleute aus dem Römischen Reich, die universi mercatores Romani imperii, eintragen lassen. ${ }^{57}$ Damit konnten die Privilegien nur gemeinsam verteidigt werden,

\footnotetext{
${ }^{49}$ Bremisches UB. I, Nr. 366, S. 404f.; ENGEL, Städtebünde (wic Anm. 34), S. 180.

${ }^{50}$ Hannoversches UB, hg. v. C. L. Grotefend und G. F. Fiedeler, Vol. I, Hannover 1860. Nr. 70, S. 66 f: : ENGEL, Städtebünde (wie Anm. 34), S. 180.

${ }^{51}$ Hannoversches UB. Nr. 79f., S. 76ff. ENGEL, Städtebünde (wie Anm. 34), S. 180.

${ }^{52}$ HUB I, Nr. 520 f., S. $181 \mathrm{f}$.

5.3 Siehe eine erweiterte Liste bei ENGEL, Städtebünde (wie Anm. 34), S. 179 f.

${ }^{54}$ Dortmunder UB, I, Nr. 413, S. 290 und Nr. 490, S. 343.

${ }^{55}$ Siehe hierzu jetzt Angela HuAng, Die Textilien des Hanseraums. Produktion und Distribution einer spätmittelalterlichen Fernhandelsware. Diss. Masch. Kopenhagen 2013.

${ }^{56} \mathrm{HR}$ I, 1, Nr. 66-72, S. 32-37.

${ }^{57}$ JAHNKE, Homines Imperii (wie Anm. 18), S. 27 ff.
} 
ungeachtet aller Streitigkeiten untereinander. Derweil war es in Flandern in den nächsten Dezennien immer wieder zu Auseinandersetzungen der Kaufleute mit der Stadt Brigge gekommen, die darin mündeten, dass zu verschiedenen Zeitpunkten der Stapel von Brügge aus in andere Städte verlegt ${ }^{58}$ oder dieses in Erwägung gezogen wurde. Die Rechtssituation in Brügge und der Zwang zur Zusammenarbeit machen deutlich, warum z. B. die wendischen Städte den engen Kontakt zu anderen Städtegruppen suchten; so z. B. am Ende des 13. Jahrhunderts, als die Städte lübischen Rechts Kontakt zu Kulm und Osnabrïck in den flandrischen Angelegenheiten aufnahmen ${ }^{59}$ oder 1305, als Lübeck Osnabrück zu einem Tag der wendischen Städte, der „civitates Slavie“, nach Wismar einlud, der die flämischen, dänischen und norwegischen Angelegenheiten behandeln sollte und zu dem sich auch Münster, Dortmund und Soest äußern sollten. ${ }^{61}$

Die Einteilung, die sich die Kaufleute in Brügge 1347 selbst gaben, zeigt die unterschiedlichen Städtegruppen in aller Klarheit an: die Kaufleute uten Romeschen rike van Almanien bilden drei Drittel: die aus dem lübischen "Ende" gehören mit denen des wendischen und des sächsischen „Endes“ in ein Drittel, die aus Westfalen und Preußen in das andere und die aus Gotland, Livland und Schweden in das dritte Drittel. ${ }^{61}$ Die Ordnung des Kontors in Brügge spiegelt sehr gut die dominierenden Städtebünde und Interessensphären der Zeit wider. Lübeck, die wendischen und die sächsischen Städte hatten schon bei der Ausschaltung des Stavens von Visby zusammengearbeitet. ${ }^{62}$ Die westfälischen Städte waren bei diesen Verhandlungen ebenfalls schon als eigenständige Gruppe in Erscheinung getreten und waren wirtschaftlich, politisch und personell eng mit den preußischen Städten verbunden. ${ }^{63}$ Die livländischen und die schwedischen, d. h. die schwedischen Städte mit einem deutsch-schwedisch geteilten Rat wie Visby, Kalmar und Stockholm, bildeten geographische Einheiten.

Allerdings war die Einteilung nicht nur ein Spiegel ihrer Zeit, sondern auch ein verdichtendes Moment, mussten die einzelnen Städtegruppen in ihren Dritteln zu Hause selbst eng zusammenarbeiten, um ihren Beitrag zur Verteidigung der immer wichtiger werdenden flandrischen Privilegien zu leisten. ${ }^{n+4}$

\footnotetext{
${ }^{58}$ U. a. HR I, 1, Nr. 21-27, S. 11-15.

${ }^{59} \mathrm{HR}$ I, 1. Nr. 77ff., S. $38 \mathrm{f}$.

60) Osnabrücker UB, Nr. 44, S. 50.

${ }^{61}$ HR I, 1. Nr. 143, S. 74-77. hier S. 75.

${ }^{62}$ Siehe unten bei Anm. 70 JAHNKE. Homines imperii (wie Anm. 18), S. $44 \mathrm{ff}$.

${ }^{6.3}$ Luise voN WinTERFELD, Das westfälische Hansequartier, in: Der Raum Westfalen, Bd. II.1, Münster 1955, S. 255-351, hier S. 284f.
}

${ }^{64}$ Siehe z. B. HR I, 1, Nr. 169 f., S. $100-103$. 
Auch in Brügge kam es zu deutlichen Separierungstendenzen. Die Lübecker sprachen konsequent von ihrem Drittel, nostra tercia ${ }^{65}$ Dieses „,nostra tercia“" wuchs bis 1356 begrifflich mit den „civitates maritimae“ zusammen. So schrieb Hamburg in diesem Jahr an seine Nachbarstädte, dass die Städte in Lübeck versammelt seien, um über Probleme in ihrem Drittel in Flandern zu beraten: Noverit vestra dilectio, quarundam civitatum maritimarum nuncios consulares, nunc l...] fuisse in Lubeke, propter causas querimoniales mercatorum in Brugis Flandrie de nostra tercia parte. ${ }^{66}$ Hintergrund dieses Schreibens waren Auseinandersetzungen am Kontor, die die Einheit - und damit die gemeinsame Privilegiennutzung gefährden könnten und die im Anschluss an dieses Schreiben in Brügge geschlichtet wurden. ${ }^{67}$ Diese Schlichtung von 1356 wurde vielfach als Ausgangspunkt der Städtehanse gesehen, da hier die Ratssendeboten aktiv in einen Streit eigentlich unabhängiger Kaufleute eingriffen. Wie allerdings die Vorgehensweise gegen den Staven von Visby gezeigt hatte, war das durchaus nichts Ungewöhnliches, so lange es einen Konsens gab.

Als zweites berührten Auseinandersetzungen mit dem norwegischen König vor allem die Handelsinteressen der wendischen Seestädte. Informiert durch die über Norwegen, besonders Bergen, nach England handelnden Kaufleute, schlossen sich 1284 Stralsund, Wismar und Rostock und später Lübeck zum Bund der „civitates maritimae“ zusammen, um gemeinsam ihre Interessen in Norwegen durchzusetzen. ${ }^{68}$ Hierbei berührten ihre Auseinandersetzungen und späteren Verhandlungen nicht nur ihre Angelegenheiten, sondern auch die der Kaufleute der Städte Stade, Osnabrück, Münster, Coesfeld, Soest, Dortmund, Leuwarden, Groningen, Staveren, Kampen, Zwolle, Deventer, Zutphen, Harderwik und Meuden. ${ }^{69}$ Die „civitates maritimae“ standen bei ihrem Vorgehen mit sehr vielen anderen Handelsstädten in Kontakt, selbst wenn sie ihren Kampf eigenständig und unabhängig durchführten.

Als drittes ist die gleiche Vorgehensweise beim Kampf der „civitates maritimae" gegen den Staven ${ }^{70}$ von Visby zu beobachten. Bis in die frühen 1290 er Jahre hinein agierten die Kaufleute im Staven frei und unabhängig im Interesse ihrer Handelsangelegenheiten. ${ }^{\text {" }}$ Allerdings berührten die von ihnen getroffenen Abmachungen die politische Interessensphäre der Seestädte im Ostseeraum direkt, so dass die Vorgehensweise des Stavens als

${ }^{65}$ HR I, 1, Nr. 163, S. $96 \mathrm{f}$.

${ }^{66}$ HR I, 1, Nr. 199, Nr. 3, S. 126.

${ }^{67}$ HR 1, 1, Nr. 200, S. $127 \mathrm{f}$.

${ }^{68}$ HR I, 1, Nr. $28 \mathrm{ff}$., S. 18f., und Nr. 34, S. 20f., 36, S. 22.

${ }^{69}$ HR I, 1, Nr. 44f., S. $25 f$.

${ }^{70}$ JAHNKE, Homines imperii (wie Anm. 18), S. $44 \mathrm{ff}$.

${ }^{7}$ Ebd. 
Bedrohung für die eigene, städtische Politik aufgefasst wurde. Die Stadt Lübeck und die mit ihr verbündeten wendischen Städte versuchten daher 1293 bis 1298, die Macht des Stavens zu brechen. Hierzu bedurften sie allerdings der Unterstützung derjenigen Kaufleute und Städte, die ebenfalls im Staven und über diesen in Novgorod präsent waren. Sie traten zuerst mit den benachbarten sächsischen Städten, den „,civitates Saxonie“, in Verhandlungen ein und teilten das erzielte Ergebnis anderen Städten dann mit der Bitte um Zustimmung mit. ${ }^{72}$ In der Folgezeit werden die „civitates maritimae“ zur treibenden politischen Kraft in diesem Raum, aus eigenem und nicht aus einem übergeordneten, irgendwie ,protohansisch" geartetem Interesse. Allerdings waren sie zur Durchsetzung ihrer Politik auf einen Konsens mit den anderen Städtegruppen angewiesen, die begannen, ihre Sendboten zu den Versammlungen der Seestädte zu schicken. ${ }^{73}$ So berichtet der Dortmunder Bote Hinrich Kale z. B. 1299 an seine Heimatstadt u. a., dass es auch den Städten gut erscheine, dass die auf Gotland, in der Gemeinschaft der Kaufleute, kein Siegel haben sollten, so dass jene nichts besiegeln könnten, was den anderen Städten nicht behage. ${ }^{74}$ Das heißt, dass die einzelnen Städte und Städtebünde, sobald es um Entscheidungen in einem größeren Raum ging, auf den Konsens und die Mitarbeit anderer Städtegruppen angewiesen waren. Heinrich Kale zum Beispiel beschreibt 1299 seinen Auftrag in einem Brief an Dortmund mit folgenden Worten: Sie seien mit den westfälischen Städten nach Lübeck gezogen, und er habe sich dort mit jenen im Namen Dortmunds eingefunden, um von den Boten der Seestädte, die sich dort zu Beratungen befanden, genaueres in Erfahrung zu bringen und vorzutragen. ${ }^{75}$ Hierdurch kam es zu einem permanenten und weit umspannenden Kontakt und Austausch zwischen regionalen und überregionalen Interessen.

\section{Die Entstehung der Hanse}

Wenn man die Vorannahme verwirft, dass es die Idee einer Hanse schon am Beginn des 14. Jahrhunderts gegeben habe, so geben die bisher vorgestellten Quellen und Ereignisse keinen Anlass zur Annahme, dass die

\footnotetext{
${ }^{72}$ HR I, I, Nr. 66-72, S. 32-37.

${ }^{73}$ Eine Übersicht über die Beschickung der Tage der Seestädte durch die Westfälischen Städte findet sich bei BERNS, Propter communem (wie Anm. 33), Tafel IV, S. 229.

${ }^{74}$ HR I. 1, Nr. 80, S. 41 f., hier S. 42. placet etiam civitatibus, quod in Gotlandia non habeatur sigillum, quod sit communium mercatorum, cum illo namque sigillari posset, quod civilatibus aliis non placeret.

${ }^{75} \mathrm{HR}$, op. cit., S. 4l. cum civitates Westfalie Lubeke pervenissent et ego vestro nomine cum eis ibidem comparuissem, civitatum maritimarum nuncios ibidem invenimus congregatos, ubi compertum est et recitatum.
} 
Verhandlungen zu Brügge 1356 etwas Großes ausgelöst hätten. Bis zu diesem Zeitpunkt können wir weiterhin die verschiedenen Städtegruppen mit ihren eigenen Interessen deutlich voneinander unterscheiden.

Allerdings waren die Gruppen in den letzten einhundert Jahren mehrmals gezwungen gewesen, zusammenzuarbeiten, um Erfolg zu haben. Und es bedurfte nur einer Initialzündung, die alle Interessen vereinen sollte. Diese Initialzündung waren die laufenden Streitigkeiten mit ausländischen Herrschern, die viele Städte und Städtebünde betrafen. Ein erstes Mal sehen wir Anzeichen hierzu im Streit mit Norwegen. Im Jahre 1343 stellte der norwegische König Magnus ein Privileg für „deutsche“ Kaufleute aus, das sich in erster Linie an die civitates maritimae wandte. Allerdings wurde im weiteren Verlauf das Empfängerfeld auf die Gemeinschaft der Kaufleute von der Hanse der Deutschen, die universi mercatores de hansa Theutonicorum, erweitert, ohne dass erläutert wurde, worin der Unterschied liegen solle. ${ }^{76}$ Allerdings wird im gleichen Jahr der Begriff weiter ausgeführt, indem der Vertreter von den Kaufleuten, die in der Hanse der Deutschen auftreten, den mercatores in hensa Theutonicorum existentes spricht. ${ }^{77}$ Hier sehen wir einen Begriff, der anscheinend unter den Verhandlungen mit Magnus gebraucht wurde, um eine Gesamtheit der Kaufleute, neben dem Begriff der "civitates maritimae“, auszudrücken. Der Urkundentext spricht im übrigen zwei Mal davon, dass die Kaufleute in der Hanse „existerunt“, d. h. für den Schreiber treten sie als Mitglieder der Hanse auf; es ist aber nicht die Rede davon, dass sie in der Hanse ,seien“.

Ähnliches sehen wir in einer der folgenden Auseinandersetzungen mit Norwegen. Die Rostocker Ratskanzlei sah sich im Laufe des Jahres 1354 wieder einmal veranlasst. Beschwerdebriefe an den norwegischen König wegen verschiedenster Konflikte zu richten. Die beiden überlieferten Entwürfe zeigen eine ganz eigene Entwicklung. Das eine Schreiben, welches Koppmann auf den 1. August 1354 datiert, spricht von unseren Bürgern und Kaufleuten, nostrorum civium et mercatorum. ${ }^{78}$ Das zweite Schreiben, vom 10. November desselben Jahres, spricht dagegen von den Kaufleuten der Hanse der Deutschen, die ihren Handel in Norwegen betreiben, mercatores de hansa Theutonicorum suas mercaciones in Norwegia exercere. ${ }^{79}$ Die Rostocker Ratskanzlei benutzte damit wie 1343 der norwegische König den Begriff der „Hanse der Deutschen“, der aus anderen Quellen nicht bekannt ist. Kannte man bisher in England die Hanse der deutschen

${ }^{70}$ HUB III. Nr. 13, S. 7f. Ich danke Dr. Volker Henn, Trier, für den Hinweis auf diese Urkunde.

${ }^{77}$ HUB III, Nr. 17, S. 10.

${ }^{78}$ HR I, I, Nr. 197, S. $123 \mathrm{f}$.

${ }^{79} \mathrm{HR}$ I, I, Nr, 196, S. $122 \mathrm{f}$. 
Kaufleute und in Brügge die Kaufleute des Römischen Reiches, sprach man nun allgemein von "der Hanse der Deutschen", deren Kaufleute mit ihren Waren nach Norwegen zogen. Hier wurde ein großer, qualitativ wichtiger Schritt vollzogen, in dem man versuchte, einen Oberbegriff für die verschiedensten Städte- und Interessengruppen, die am Handel in Norwegen - und in der Folge auch andernorts - interessiert waren, zu finden. Dass man sich hierzu des geographisch und politisch unbestimmten Begriffes „der Hanse der Deutschen" bediente, kann auch daran gelegen haben, dass die im 13. Jahrhundert in England und Flandern gewählten politischen Begriffe, z. B. der Kaufleute des römischen Reiches, als Nebenwirkung u. a. die preußischen und livländischen Kaufleute ausgeschlossen hatten. ${ }^{80}$ Hier hingegen war der Begriff der „Hanse der Deutschen" vage genug, um alle Gruppen mit zu umfassen, zumal das Recht des ,gemenen kopmans“, dem sie angehörten, ja das einzige verbindende Element zwischen den einzelnen Gruppen war.

Es sollte allerdings vier Jahre brauchen, bis sich der Begriff wirklich durchgesetzt hatte. Anlass war die Verletzung der Privilegien in Brügge und der Gedanke, einen Boykott des flandrischen Handels zu beschließen. Um den hierzu notwendigen Konsens zu erzielen, hatte der Rat von Lübeck Vertreter des preußischen Drittels sowie Goslar als Vertreter der sächsischen Städtegruppe zu Verhandlungen nach Lübeck geladen. Der auf dieser Versammlung ausgearbeitete Rezess ${ }^{81}$ spricht, wie schon Thomas Behrmann ${ }^{82}$ und Stephan Selzer gezeigt haben, ostentativ von "der deutschen Hanse“, und nicht mehr von ,der Hanse der Deutschen". Von den 12 Artikeln nennen sieben die Hanse explizit: so zum Beispiel Artikel 1: kein Bürger und niemand aus der deutschen Hanse soll nach Flandem fahren oder Kapitel acht: alle Kaufleute aus Deutschland (Alemanien), die in der deutschen Hanse sind, sollen Flandern verlassen. Allein der letzte Artikel zeigt, wie sehr der neue Begriff noch einer Erklärung bedurfte, waren die Kaufleute aus Alemanien ja diejenigen, die das Privileg von 1252 erhalten und verteidigt hatten. Der neu gefundene Begriff wird dann auch am gleichen Tag den Städten der Drittel mitgeteilt, die nicht in Lübeck vertreten waren, so zum Beispiel Dortmund, dem mitgeteilt wurde, was die ehrenwerten Herren, die Ratsherren der Städte unseres Drittels der Kaufleute des Königs der Römer aus Deutschland aus der Hanse der Deutschen, die nach Brügge in Flandern ziehen, beschlossen hatten. ${ }^{87}$ Hier

\footnotetext{
${ }^{80}$ JAHNKE, Homines imperii (wie Anm. 18).

${ }^{81}$ HR I, I, Nr. 212, S. $135 \mathrm{ff}$.

82 Thomas Behrmann, Herrscher und Hansestädte. Studien zum diplomatischen Verkehr im Spätmittelalter (Greifswalder Historische Studien, Bd. 6), Hamburg 2004. S. 16 f.

${ }^{80} \mathrm{HR}$ I, 1, Nr. 213, S. $137 \mathrm{ff}$. honorabili viri, domini consules civitatum (...) de nostra
} 
haben wir die Geburtsstunde des Hansebegriffes vor uns, eine Geburtsstunde, die nicht alle späteren Hansestädte erkannten. So beschwert sich z. B. Groningen darüber, nicht informiert worden zu sein, und verwendet dabei eben nicht den Begriff der Hanse, sondern den der Dusken stede. ${ }^{84}$ 1358 war der Begriff eingeführt worden. es brauchte aber seine Zeit, bis er fest implementiert war. So verwendete zum Beispiel Bremen, das gegen die Vereinbarungen vom Januar verstoßen hatte und aus den Privilegien ausgeschlossen worden war, in seiner Sühneurkunde den Begriff der Ratsherren der Seestädte und der anderen Städte, sowie der Gemeinschaft der Kaufleute der Hanse der Deutschen des Heiligen Römischen Reiches, consules civitatum maritimarum et etiam aliarum civitatum, necnon communes mercatores de hansa Theutonicorum sacri Romani imperii, ${ }^{85}$ ein mehr als deutlicher Pleonasmus, der die begriffliche Unsicherheit der Bremer Kanzlei sehr gut zum Ausdruck bringt. Die Empfänger des Sühneschreibens werden gleich in dreierlei Funktion angesprochen, als die Seestädte des wendischen Drittels „civitates maritimae“, als Vertreter der Gemeinschaft der Hanse der Deutschen, die wiederum mit Reminiszenzen an die alte Bezeichnung der Kaufleute in Brügge versehen wird. Das zeugt nicht davon, dass man in Bremen im Sommer 1358 genau wusste, was die deutsche Hanse eigentlich war. Das zeigt vielmehr einen Begriff im Entstehen.

Die Implementierung des neuen Begriffes bedeutet nun auch nicht, wie die Hanserezesse vermitteln möchten, dass alle kommenden Versammlungen „hansisch“ gewesen seien oder die Hanse als feste Institution das Licht der Welt erblickt habe. Als Beispiel sei nur darauf hingewiesen, dass sich die wendischen Städte, die sich im November 1358 in Greifswald trafen, als die Städte, die sich auf dem lïbischen Recht gründen, civitates quia cum jure Lubicensi fundati [sunt], bezeichnen. ${ }^{86}$ Die alten Merkmale des Zusammenhaltes wurden durch den neuen Begriff der „Hanse“ natürlich nicht aufgehoben, sondern sollten in der neuen Struktur fortleben. Auch ist es schwer, Karl Koppmann zu folgen, wenn er die nächste Versammlung der Städte in Sachen des Kontores von Brügge im Juni 1359 pauschal als „Hansetag“ bezeichnet. Im Einladungsschreiben werden ausdrücklich zuerst nur die „civitates maritimae“ eingeladen, von einer ,hansischen" Angelegenheit kann also nicht die Rede sein, ${ }^{87}$ auch, wenn die

\footnotetext{
tercia parte omnium mercatorum regis Romanorum de Alemannia de hansa Teutonicorum in Brugis Flandrie moram trahencium.

${ }^{84}$ HR I. I, Nr. 214, S. 139.

${ }^{85}$ HR I, I, Nr. 216, S. $143 \mathrm{f}$.

${ }^{86}$ HR I, I, Nr. 220, S. $146 \mathrm{f}$.

${ }^{87}$ HR I. 1, Nr. 224, S. $151 \mathrm{f}$.
} 
märkischen Städte zu dieser von den Ratsherren der „civitates maritimae“ einberufenen Versammlung eingeladen werden. ${ }^{88}$ Doch weitet sich der Kreis der Eingeladenen weiter aus, und im abschließenden Rezess wird dann, sehr konservativ, von den Ratsherren der deutschen Städte des Römischen Reiches, die in Lübeck versammelt sind, consulares civitatum Almanie regni Romanorum congregatos in Lubeke, gesprochen. ${ }^{89}$ Auch bei den anschließenden Verhandlungen in Flandern wird der Begriff der Hanse nicht gebraucht, sondern konsequent von den Kaufleuten aus Almanie gesprochen. ${ }^{90}$

Ebenso unterließen es ausländische Herrscher, den neuen Begriff zu nutzen. So sprach Waldemar Atterdag 1360 die Städte mit dem Begriff der Seestädte aus dem südlichen Teil [der Ostsee], civitates maritimae partium australium, an, ${ }^{91}$ was wiederum davon zeugt, dass sich der Hansebegriff nicht etabliert hatte.

Doch scheinen die Abgesandten der Städte mehr und mehr darauf bestanden zu haben, dass der neue Begriff verwandt wird. Sprachen so z. B. Graf Ludwig von Flandern und die Stadt Ypern im Juli 1360 noch von den Kaufleuten aus „Almanie, ${ }^{92}$ so bezeichnen sich die Boten der Städte im August des Jahres selbst als boden to den menen steden des kopmannes van der Dudeschen hense. ${ }^{93}$ Andererseits werden die ersten Anfänge des Krieges gegen Dänemark 1361 noch von den Städten by der zee, d. h. den „civitates maritimae“ und nicht der deutschen Hanse eingeleitet, ${ }^{94}$ die gefassten Beschlüsse werden aber im Namen der hansa Theutonicorum durchgeführt. ${ }^{95}$ So lädt Lübeck zum Beispiel Reval zu einer Tagung der Ratsherren der Seestädte, consulares civitatum maritimarum, ein, die mit den Abgesandten des Hochmeisters und der preußischen Städte stattfinden solle, und bei der u. a. die Frage im Raum stand, wie man Schonen und Dänemark boykottieren könne, so dass diese von niemandem aus der Hanse der Deutschen mit seinen Waren besucht werden. ${ }^{96}$ Es handelt sich also um Angelegenheiten, die die Kaufleute in ihrer Gesamtheit betreffen und

\footnotetext{
${ }^{88}$ HR I, 1, Nr. 225, S. $152 \mathrm{f}$.

${ }^{89}$ HR I, 1, Nr. 227, S. 155 f., hier S. 156.

${ }^{90}$ HR I, 1, Nr. 228-231, S. 157-160.

${ }^{91}$ HR I, 1, Nr. 232, S. $162 \mathrm{f}$.

${ }^{92}$ HR I, 1, Nr. $237 \mathrm{ff} .$, S. $168 \mathrm{ff}$.

${ }^{93}$ HR I, 1, Nr. 251, S. 177-180, hier S. 177 und passim.

${ }^{94}$ HR I, 1, Nr. 259f., S. 186-190. Liv-, Esth- und Kurländisches UB, I.2, Nr. CMLXXXVII, Sp. 694-697.

${ }^{95}$ HR I, 1, Nr. 264, S. $192 \mathrm{ff}$.

${ }^{96}$ Liv-, Esth- und Kurländisches UB, 1.2, Nr. CMLXXXVII. Sp. 694-697, hier Sp. 695. Quod debeat regnum et dominium Daciae et terra Schaniae evitari, nec per aliquem de hansa Theutonicorum cum aliquibus bonis visitari, hoc tamen observando sub amissione corporis et rerum et poena.
} 
damit „hansisch“ waren. Allerdings sind es die einzelnen Städtegruppen, die ihre Interessen einbringen sollten. Es sind aber auch die ,civitates maritimae", die im Folgenden als Einheit tagen, ${ }^{17}$ wenn auch wiederum der Waffenstillstand zwischen der Hanse und Dänemark abgeschlossen wurde. ${ }^{98}$

In der Zwischenzeit hatte der neue Begriff aber auch einen neuen institutionellen Inhalt erfahren. Im Zuge des Krieges hatten die Seestädte und die preußischen Städte (tam maritimis quam Prutiae) ${ }^{99}$ zur Deckung der Unkosten einen Pfundzoll erhoben. Hiermit erhielt das Bündnis zur Verteidigung der Privilegien eine neue Dimension und eine neue Bedeutung. Allerdings muss auch darauf hingewiesen werden, dass "hansisch“ in Bezug auf diesen Pfundzoll nur als Beitrag der Preußen und der beteiligten Seestädte zu verstehen ist. ${ }^{101}$ Die „Hanse“, die im Kampf gegen Norwegen und in Flandern aufgetreten war, ist hier nicht präsent. ${ }^{101} \mathrm{Al}-$ lerdings betrachteten sich die civitates wie die preußischen Städte durchaus als Vertreter eines wie auch immer gearteten gesamthansischen Interesses, wenn sie feststellen, dass diejenigen, die in der Hanse der Deutschen seien, und den vereinbarten Zoll nicht geben, aber Dänemark und Schonen besuchen wollten, zu boykottieren seien. ${ }^{102}$ In den folgenden Jahren bis 1368 sind es dann auch vornehmlich die "Seestädte“, die in den Verhandlungen mit Waldemar Atterdag auftreten. Die im Bündnis vereinigten Städte selbst betrachten sich zudem als Gemeinschaft der Seestädte, communes civitatae maritimae, und nicht als Hanse. ${ }^{10^{3}}$

Auch bei der von den Preußen und Holländern in Gang gebrachten Konföderation von Köln können wir ähnliche Verschiebungen in der Selbstbezeichnung feststellen. Sind die ersten von der Kölner Versammlung ausgehenden Schreiben noch mit „Ratsherren der Seestädte, die zur Zeit in Köln versammelt sind", consules civitatum maritimarum pronunc Colonie congregatos unterzeichnet, ${ }^{104}$ so ändert sich der Titel in ,Ratsherren und Boten der Städte der deutschen Hanse, die zur Zeit in Köln versammelt sind", Consules nuncii civitatum de hanza Theotonica

\footnotetext{
${ }^{97}$ HR I. 1, Nr. 267, S. $200 f$.

${ }^{98} \mathrm{HR}$ I, 1, Nr. 277, S. 206-210 (Entwurf); das ausgefertigte Original UBStL 3, Nr. 440.

${ }^{99}$ Liv-, Esth- und Kurländisches UB. 1.2, Nr. CMLXXXVII, Sp. 694-697, hier Sp. 695.

${ }^{100}$ Liv-, Esth- und Kurländisches UB, I.3, Nr. MXLVIII, Sp. $206 f$.

${ }^{101}$ HR I, I, Nr. 280, S. $210 \mathrm{ff}$.

${ }^{102}$ Liv-, Esth- und Kurländisches UB, I.2, Nr. CMLXXXVII. Sp. 694-697, hier Sp. 696. similirer si qui essent in hansa Theutoniconum, qui huiusmodi theolonium dare nollent, et dicta. scil. regna Daciae et Schaniae vellem visitare, cum illis nullus emere nec vendere debet.

${ }^{103}$ Liv-, Esth- und Kurländisches UB, 1.2, Nr. MXIII, Sp. 727.

${ }^{104}$ HR 1, I, Nr. 414f.. S. $376 f$.
} 
pronunc in Colonia congregati, ${ }^{105}$ um dann wieder zurück in die alte Bezeichnung zu wechseln. ${ }^{106}$ Dieser Wechsel in der Selbstbezeichnung ist ein Ausdruck der verschiedensten Interessen und Handlungsrichtungen. Es wird deutlich, dass zwischen agierenden Städtebünden und einem ,hansischen" Interesse unterschieden werden konnte.

Es ist allerdings zu fragen, was in dem Krieg gegen Dänemark „hansisch" hätte sein können, betraf er ja die Interessen vieler Binnenstädte nicht. So verhielten sich z. B. die sächsischen Städte wie eine Vielzahl anderer Städte nach Ansicht vieler moderner Historiker passiv, womit implizit angedeutet wird, dass sie eine wie immer geartete Verpflichtung zum Handeln gehabt hätten. ${ }^{107}$ Insofern kann an dieser Stelle konstatiert werden, dass es schon reichte, wenn einige Städtegruppen teilweise Interessenüberschneidungen besaßen, um eine Angelegenheit als „,hansisch" zu bezeichnen. Die Intentionen und die Ziele der Teilnehmer der Kölner Konföderation waren ja durchaus unterschiedlich, gemeinsam war ihnen aber, dass ihre Kaufleute und ihr Handel im Ostseeraum resp. am Öresund betroffen waren. Ein wie auch immer geartetes eigenständiges ,gesamthansisches" Interesse kann aber ausgeschlossen werden, da es ein solches nicht gegeben hat.

Es wird deutlich, dass selbst in der Zeit um 1368 die Hanse als Institution noch nicht fertig ausgeprägt war, wie aus einem Schreiben der Seestädte nach Brügge vom 2. Februar des Jahres hervorgeht in welchem die Ratsherren der Seestädte, die in Lübeck versammelt seien, die Älterleute und den gemeinen Kaufmann der deutschen Hanse in Brügge grüßen und erklären, dass die Seestädte mit den preußischen Städten, Kampen, den seeländischen und holländischen vereinbart hätten, dass niemand, der in der Hanse sei, durch den Sund in die Westsee segeln solle. ${ }^{108}$

Die Seestädte verhandeln also mit anderen Städten ihres Drittels sowie mit preußischen, den seeländischen und holländischen Städten und teilen dieses den Älterleuten der deutschen Hanse in Brügge mit, wobei die Beschlüsse wiederum ,hansisch“ werden konnten. Der Hansebegriff wird hier zwar verwandt, allerdings ist es nicht „die Hanse“, die hier eine Ent-

\footnotetext{
${ }^{105}$ HR I, 1, Nr. 416, S. $377 \mathrm{f}$.

${ }^{106}$ HR I, 1, Nr. 4l8f.. S. $378 \mathrm{ff}$.

${ }^{107}$ PuHLE, Sächsischer Städtebund (wie Anm. 43), S. 20.

${ }^{108}$ HR I, 1. Nr. 428, S. 388f, hier S. 388. Prudentibus ac honestis viris, aldermannis et communi mercatori hanse Theotonice Brugis Flandrie existentibus, nostris amicis dilectis presentetur. Consules civitatum maritimarum in data presencium Lubeke congregati previa salucione amicabili decenti [...J. Noveritis, quod nos consules civitatum Lübeke, Rostock, Stralesund et Wismar una cum aliis civitatibus nostrarum parcium concordavimus cum civitatibus Prucie, Campen, Selandie et Hollandie sic: quod nullus nostratum in hansa existens (...) volens velificare per portum Sund versus mare orientale.
} 
scheidung trifft, sondern es sind die alten Städtegruppen, die als treibende Kräfte fungieren. ${ }^{109}$ So berichtet z. B. im Februar 1368 der rigische Rat nach Reval, dass ,sich Eure Boten", die nuntii vestri de nuntiis civitatum huius patrie in Pernau getroffen hätten, um über die Entwicklungen des Krieges zu beraten. U. a. kam zur Sprache, dass der revalsche Ratsherr Herman de Hove aus Preußen berichten konnte, dass die Gotländer nicht zu den Dänen, sondern zu den „Kaufleuten der Deutschen“, mercatores Theutonici, halten würden. ${ }^{110}$ Es gab noch keine Struktur, die die Hanse als Institution widerspiegeln würde, und es ist damit die Frage, ob man von ,der Hanse“ als einer bestehenden Institution zu diesem Zeitpunkt überhaupt schon sprechen kann. Diese Zweifel werden noch bestärkt, schaut man sich den offiziellen Klagebrief der Städte gegen Valdemar Atterdag an. In diesem Schreiben, gerichtet an verschiedene Fürsten und Herren, bezeichnen sich die Städte selbst nur als „Gemeinschaft der Kaufleute", communes mercatores, und nicht als Hanse. ${ }^{11}$ An dieser Stelle kann argumentiert werden, dass es klüger war, nicht als (durch die Goldene Bulle verbotener ${ }^{12}$ ) Städtebund aufzutreten, doch hat Karl IV. die Hanse niemals als solchen angesehen, ${ }^{113}$ so dass der Begriff unverfänglich erscheinen musste.

Andererseits treten die Städte in toto, aufgeführt mit Namen und versehen mit dem Zusatz de in der Dudeschen hense sint als Vertragspartner König Albrechts von Schweden gegen Dänemark auf. ${ }^{114}$ Ebenso sprechen die Stadt Dordrecht und Albert von Bayern von den Kaufleuten aus „Almanie" und denen von der deutschen Hanse (coplude van Alemangen ende van der Dutscher ansen) als zwei Gruppen, die nicht identisch sind. ${ }^{115}$

Darüber hinaus betrachten die Städte selbst die Kölner Allianz nur als eine zeitweilige Konföderation, die einen zielgerichteten und zeitlich begrenzten Charakter besaß. So war eines der Themen der Lübecker Tagfahrt von 1384, ob man das Bündnis und die Liga, die man bisher untereinander gehabt habe, verlängern solle, de confoederatione et liga, quam civitatenses invicem habuerunt, an ipsam velint prolongare, an permittere

${ }^{109}$ Siehe zum Gedanken der Teilräume als bestimmende Elemente innerhalb der Hanse schon Volker HENN, ,... de alle tyd wedderwartigen Suederseeschen stedere“. Zur Integration des niederrheinisch-ostniederländischen Raumes in die Hanse, in: HGbll. 112, 1994, S. 39-56, hier S. $40 \mathrm{f}$.

${ }^{110}$ Liv-, Esth- und Kurländisches UB, 1.3, Nr. MXLVI, Sp. 204.

11 HR I, 1, Nr. 431, S. $390 \mathrm{ff}$, hier S. 390.

${ }^{112}$ Bulla Aurea Karoli IV. Imperatoris Anno MCCCLVI promulgata, hg. v. Wolfgang D. FrITZ, MGH, Font. lur. Germ., Vol. XI, Weimar 1972, § XV, S. 70 f.

${ }^{113}$ Heinz STOOB, Kaiser Karl IV. und der Ostseeraum, in: HGbll. 88, 1970, S. 163-2 14. hier S. $177 \mathrm{f}$.

${ }^{134}$ HR I, I, Nr. 453, S. 410-413.

${ }^{115}$ HR I, 1, Nr. $450 f .$, S. $404 \mathrm{ff}$. 
resolutum manere. ${ }^{116}$ Die Kölner Konföderation war somit nichts anderes, als ein zeitlich begrenztes Bündnis.

Ein ähnliches Bild erhalten wir, wenn wir uns die Auseinandersetzung der Städte mit den Aufständischen anschauen, die 1374 den Rat in Braunschweig vertrieben hatten. Der Rezess der ,civitates maritimae“ vom 21. Mai 1374 behandelt in $\$ 6$ den Aufstand in Braunschweig: Die Städte drohen Braunschweig an, deren Kaufleute aus dem Recht der Kaufleute auszustoßen. ${ }^{177}$ In dem anschließenden Rezess vom 25 . Juli wird berichtet, dass die Städte einen Brief in dieser Angelegenheit gesandt hätten van den menen steden den steden in Lyvlande, in Prucen, in der Marke, in dem lande to Sassen, to Colne, in Wistphalen, to Bremen, to Staten, unde to alle den steden, de in des copmans rechte sind $;^{118} \mathrm{~d}$. h., die Städte, vertreten durch die Städtegruppen, drohen Braunschweig, i. e. eigentlich den Kaufleuten der Stadt, sie von dem Genuss der Privilegien außerhalb auszuschließen. In diesem Zusammenhang erscheint die Hanse nicht. Es war kein Hansetag, der die Sache behandelt hat, sondern die Städtegruppen behandeln Dinge, die einen hansischen Charakter erhalten, da sie Rechte betrafen, die die Städte zusammen erworben und verteidigt hatten und die jetzt von den explizit genannten Vertretern einzelner Städtegruppen verhandelt worden waren. Dieses wird auch in der daran folgenden, direkten Auseinandersetzung mit den Aufständischen in Braunschweig deutlich. Die Städte schreiben der Stadt Braunschweig im Namen der gemeinen Städte, die in der Hanse der Deutschen versammelt sind, nomine universarum civitatum in hensa Theotonicorum comprehensarum. ${ }^{119}$ woraufhin sich die braunschweigischen Gilden darüber beschweren, dass die ratheren [von Lübeck, CJ] mit den anderen raaden der seestede übereingekommen wären, Braunschweig zu verfolgen. ${ }^{120}$ Lübeck und die Seestädte versuchen, durch ihr Vorgehen einen allgemeinen, regionenübergreifenden Konsens zu schaffen, wohingegen die Braunschweiger in ihren Taten nur eine Verfolgung durch eine benachbarte Städtegruppe sehen. Dass diese Auffassung nicht völlig aus der Luft gegriffen war, bezeugt ein weiteres Schreiben in dieser Angelegenheit. Am 24. Juni 1375 wird Minden mitgeteilt, dass Braunschweig nun wirklich ute der hense unde utes copman-

\footnotetext{
${ }^{116}$ Liv-. Esth- und Kurländisches UB. 1.3, Nr. MCCXVI, Sp. 4411.

${ }^{117}$ HR I, 2, Nr. 73, S. 81 ff., hier S. 82.

${ }^{118}$ HR I, 2, Nr. 77, S. 86-89, hier S. 86.

${ }^{119}$ HR I, 2, Nr. 82, S. 94.

${ }^{120}$ HR I, 2, Nr. 84, S. 95f., hier S. 95. Diese Argumentation war sicherlich der Versuch der Braunschweiger Aufständischen, einen Keil in die Gruppe ihrer Gegner zu treiben. Allerdings zeigt das Vorspiel in Lübeck, dass die Argumente der Braunschweiger durchaus nicht der Logik entbehrten. Ich danke Prof. Matthias Puble in Magdeburg für den Hinweis auf die besonderen Braunschweiger Verhältnisse.
} 
nes rechte unde vryhed verstoßen worden sei. Unterzeichnet ist das Schreiben aber nicht von den „Hansestädten“, sondern von den Ratsherren der Seestädte, wie auch der preußischen, livländischen und mittelmeerischen Städte, die zur Zeit in Lübeck versammelt seien, den [consulibus] civitatum maritimarum, necnon Pruscie, Lyvonie et de Mare meridiano in data presencium Lubeke ad placita congregati. ${ }^{121}$

In den Augen der Braunschweiger - und auch nach Aussage der Quellen - ist die Hanse nur ein ad hoc benutzter Begriff, wenn es um Angelegenheiten von übergeordneter Bedeutung geht. Die Versammlungen der Städte waren dabei explizit nicht als „Hansetagungen“ angelegt, sondern als regionale Städtetage, die auch andere Angelegenheiten behandeln konnten. Alle Rezesse, die Koppmann bis zum Jahr 1379 gesammelt hat, beginnen mit der Standardformel „Wir Ratsherren/Boten der Seestädte, versammelt in der Stadt NN“, ,consules/nuncii consulares civitatum maritimarum congregati in $\mathrm{NN}^{\text {“ }}$, und sind damit keine Aufzeichnungen eines „Hansetages“. Zwar kam es nach 1368 zu einer Häufung der Städtetage, auf denen jetzt mehr und mehr auch die seeländischen, holländischen oder die preußischen Städte vertreten waren, aber ,gesamthansisch“ waren diese Versammlungen nicht.

Hierbei können die Bezeichnungen auch wechseln. So schreibt am 23. Mai 1376 das Kontor in Brügge an die erbare[n] [...] heren borghermesteren unde ratluden in der dachvart to Stralsunde van der Dudeschen hense unde der ghemeynen zeesteden, ${ }^{122}$ die sich selbst aber wiederum nur als Ratssendeboten der in Stralsund versammelten Seestädte, nuncii consulares civitatum maritimarum congregati in Stralessundis bezeichnen. ${ }^{123}$ Ebenso verwenden größere Verträge, die mehrere Städtegruppen umfassen, nicht den übergeordneten Hansetitel, sondern Regionalbezeichnungen. So wird der 1376 mit König Håkon von Norwegen geschlossene Frieden an die Seestädte, civitates maritimae sowie die livländischen, preußischen, zuiderzeeischen, zeeländischen, holländischen und gelrischen Städte, civitates Lyvonie, Pruscie, de Mari australi, Zelandie, Hollandie, Ghelrie, sowie Staveren und Hindeloep, die in, jenem Recht und Bündnis versammelt sind", ipsarum jure et confederacione comprehensarum [sunt], gerichtet. Der Begriff „Hanse“ erscheint nicht. ${ }^{124}$

Allerdings wird in den von Koppmann gesammelten Dokumenten auch eine Tendenz deutlich, die institutionelle Rolle der Städtegruppen über die Friedenssicherung und Privilegienbewahrung hinaus auszudehnen. Bei-

\footnotetext{
${ }^{121}$ HR I, 2, Nr. 92f., S. $106 f .$, hier Nr. 93, S. 107.

${ }^{122}$ HR I, 2, Nr. 119, S. I29f., hier S. 129.

${ }^{123}$ HR I, 2, Nr. 120, S. $131 \mathrm{f}$, hier S. 131.

${ }^{124}$ HR I, 2, Nr. 124, S. $136 \mathrm{ff}$, hier S. 136.
} 
spiele hierfür sind u. a. der Städtetag vom 24. Juni 1375 in Lübeck, der einheitliche Maße für Pech-, Teer-, Asche- und Heringstonnen festlegte, ${ }^{125}$ sowie der vom 23. März 1376 in Stralsund, der die Qualität von Kannen und Grapen festlegte. ${ }^{126}$ Die Schifferordnung von $1378 .{ }^{127}$ oder die Münzunion von 1379 sind weitere Beispiele. ${ }^{128}$ Alle Beschlüsse sind von den „civitates maritimae“ getroffen worden, haben also einen regionalen Charakter, betreffen in ihren Auswirkungen aber einen ungleich größeren Handelsraum. ${ }^{129}$

Beide Entwicklungen zusammen erklären eine Diskrepanz, die bisher in der Forschung zwar vielfach gesehen, aber nur schlecht erklärt werden konnte: die mangelnde Teilnahme eines großen Teiles der „Hansestädte“ an den politischen Aktionen dieser Jahre. Im Allgemeinen wird konstatiert, dass sich bestimmte Städtegruppen, z. B. die märkische, sächsische oder die westfälische, passiv verhalten hätten. Nach der oben dargestellten Entwicklung waren sie bis dahin aber gar nicht beteiligt und schon gar keine „Hansestädte" im bisherigen Verständnis. Eine Zusammenarbeit war notwendig, wenn es um Angelegenheiten gemeinsam besuchter Räume, wie der späteren Kontore, ging. Da hier die Vorgehensweise nur im Konsens erfolgen konnte, musste man eine gemeinsame Hanse bilden. Wenn es aber nur regionale Angelegenheiten betraf, entschied und verhandelte man auch nur regional. ${ }^{130}$ Die von Koppmann getroffene Auswahl und vor allem seine Bezeichnung der Rezesse als Rezesse der Hansetage sind daher in diesem Zusammenhang nicht nur irreführend, sondern definitiv falsch.

Ebenso falsch ist es, die Entstehung der Hanse allein in Lübeck und bei den wendischen Städten zu sehen. Die Konzentration auf die Seestädte beruht einzig und allein auf die von Koppmann getroffene Auswahl, wie die HR I, 2, Nr. 174ff, zeigen. In diesen Akten werden Vorgänge aus Preußen überliefert, die in ihrer Anlage, Struktur und Ausformung denen der wendischen Städte gleichen. In diesem Bereich ist daher noch sehr viel mehr Forschung notwendig, um die regionalen Zusammenhänge besser zu verstehen.

\footnotetext{
${ }^{125}$ HR I, 2, Nr. 86, S. 98-102, hier $\$ \$ 12$ f., S. 101 .

${ }^{126}$ HR I, 2, Nr. I15. S. 123 f., hier $\$ \S$ If., S. 123.

${ }^{127}$ HR 1, 2, Nr. 157, S. 171 .

128 HR I, 2, Nr. 172, S. $187 \mathrm{f}$.

${ }^{129}$ Die civitates waren aber nicht die Einzigen, die eine solche institutionelle Erweiterung vollzogen hatten. So haben auch die sä̀chsischen Städte 1382 eine Münzkonvention vereinbart. HENN. Städtebünde (wie Anm. 41). S. 58. HR I. 3, Nr. 154, S. 133 f.

${ }^{130}$ Siehe zu diesem Gedanken schon Ahasver VON BRANDT, Die Hanse und die nordischen Mächte im Mittelalter. Wiederabdruck in: Lübeck. Hanse, Nordeuropa, Gedüchtnisschrift für Ahasver von Brandt, hg. v. Klaus Friedland und Rolf Sprandel, Köln 1979. S. 13-36. hier S. $16 \mathrm{f}$.
} 
VII. Wann entstand also die deutsche Hanse als Institution und wann wurde aus ihr eine Organisation?

Der Entwicklungsprozess der Hanse seit 1350 erreichte einen deutlich sichtbaren Scheidepunkt im Zeitraum 1379/80. Zu dieser Zeit wurde ein Institutionalisierungsschub und damit eine qualitative Aufwertung der Hanse manifest, vor allem, wenn man die beiden Versammlungen vom Juni 1379 und August 1380 in Lübeck betrachtet.

Sieht man sich die Lübecker Versammlung von 1379, die am 24. des Monats mit einem Rezess abgeschlossen wurde, ${ }^{131}$ an, so unterscheidet sich der Rezess als solcher kaum von den vorhergehenden: Flandern und Dänemark stehen im Fokus, und es wird eine Verordnung über Dienstknechte erlassen. Aber die begleitenden Umstände lassen aufhorchen. Schon in der Einladung vom 30. März 1379, die u. a. den von allen Seestädten, omnibus civitatibus maritimarum, erhobenen Pfundzoll behandelt, werden die Städte zu einem Tag der ,gemeinen Städte“, der communes civitates, in Lübeck eingeladen, der Flandern, England und andere Angelegenheiten behandeln sollte. ${ }^{132}$ Die bremische Chronik von Rynesberch berichtet zu diesem Tag: Do die Hamborgher aldus rike wurden van ereme bere, unde begunden to stighende in rikedaghen, do wolden sie ock stighen boven den rad unde de stad van Bremen mit vorgaande, dar die menen hentzestede thosamende plegen to komende, des sie oldinges ny gedan en hedden. ${ }^{133}$ Im Zusammenhang mit diesem Tag kam es also zu Unstimmigkeiten im zeremoniellen Rang zwischen diesen beiden Städten, etwas, das eine feste Ordnung, um die man kämpfen konnte, voraussetzt. Dabei war es kein Zufall, dass Hamburg und Bremen genau zu diesem Zeitpunkt über den Vorrang auf dem „Hansetag“ stritten: es deutet alles darauf hin, dass die Städtegruppen genau auf dieser Versammlung Schritte zu einer Institutionalisierung der Hanse einleiteten. So beginnt der Rezess noch ganz traditionell mit den Worten: Im Jahre des Herren 1379, am Tage des Heiligen Johannes des Täufers, waren in Lübeck die Herren Ratssendboten der Seestädte und anderer, nachfolgend aufgeführter Städte versammelt. ${ }^{134}$ Aber schon im nächsten Rezess wurde die Einleitungsformel an einer entscheidenden Stelle wesentlich verändert. Der Rezess des Tages vom 12. August 1380 beginnt, wie die nachfolgenden, mit den Worten: in

\footnotetext{
${ }^{131}$ HR I, 2, Nr. 190), S. 207-211.

${ }^{132}$ Liv-, Esth- und Kurländisches UB, I.3, Nr. MCXXXVI, Sp. $341 \mathrm{f}$.

${ }^{133}$ HR 1, 2, Nr. 189, S. 205 f., hier S. 205.

${ }^{134}$ HR I, 2, Nr. 190. S. 207-211, hier S, 207. Anno Domini [1379] in festo nativitatis sancti Johannis baptiste Lubeke ad placita congregati domini nuncii consulares civitatum maritimarum et aliarum infrascriptarum.
} 
Lübeck sind die Ratssendboten der folgenden Städte versammelt, in $\mathrm{Lu}$ beke congregati [sunt] consulares nuncii infrascriptarum civitatum, ${ }^{135}$ gefolgt wie z. B. auch am 21. Oktober in Wismar ${ }^{136}$ von einzelnen Städten oder aber Städtegruppen, wie den civitates maritimae oder Pruczie. Eine gleiche Tendenz findet sich in den folgenden Einladungsschreiben wieder. So lädt Lübeck am 22. Juli 1383 Riga zu einem Tag der gemeinen Städte, der communes civitates, nach Lübeck ein, zu dem die civitates Prutzie auch ihre Boten entsenden und den die livländischen Städte auch beschicken wollten. ${ }^{137}$ Die ,civitates maritimae“ treten als Gruppe also zurück und sind nur noch ein Teil einer Versammlung, die einen mehr allgemeinen Charakter angenommen hat. Aus der regionalen Versammlung der Seestädte, die ad hoc auch allgemeine, „hansische“ Angelegenheiten behandeln konnte, wurde die Versammlung der gemeinen Städte, die in Gruppen organisiert und von Gruppen repräsentiert, auf diesem Tag erscheinen konnten - wenn es in ihrem Interesse lag. Hiermit wurde die Grundlage für die organisatorische Ausbildung der Hanse gelegt. Aus dem sich nur bei gewissen zeitlich begrenzten Vorgängen zeigenden Zusammenhalt der Hanse wurde 1379 ein eigener Städtetag, eine feste Institution, die sich erst auf dieser Grundlage weiter entwickeln konnte. Der zeremonielle Aspekt des Vorrangstreites zwischen Hamburg und Bremen sowie die symbolische Veränderung des Recessformulares ${ }^{138}$ sind dabei sichere Indizien für die institutionelle Veränderung der Hanse gerade zu diesem Zeitpunkt.

Die Hanse war am Ende des 14. Jahrhunderts eine Versammlung, zu der die Städtegruppen erscheinen konnten, wenn sie wollten und wenn sie einen Erfolg durch gemeinsames Handeln erwarteten. Hanse institutionalisierte sich aber nur dann, wenn es gemeinsame Ziele gab. Sie existierte zu diesem Zeitpunkt nicht aus sich selbst heraus, sie war noch keine Organisation.

Der oben aufgezeichneten Entwicklung lagen wahrscheinlich verschiedene Ursachen zugrunde. Dass es ausgerechnet die wendischen Städte waren, deren Tage zum Forum für allgemeine Fragen wurden, lag vermutlich darin begründet, dass Lübecker und Hamburger Kaufleute auf-

\footnotetext{
${ }^{135}$ HR I, 2, Nr. 219, S. 261 f. Siehe diesen Gedanken in Ansätzen schon bei BEHRMANN, Herrscher und Hansestädı (wie Anm. 82), S. 19.

${ }^{136}$ HR I, 2, Nr. 220, S. 263-268, hier S. 263.

${ }^{137}$ Liv-, Esth- und Kurländisches UB, I.3, Nr. MCXCIIf., Sp. 405-409.

${ }^{138}$ Siche zur Entwicklung des Rezessformulares, zu dessen symbolisch-institutioneller Relevanz und mit sehr ähnlichen Ergebnissen wie die vorliegende Studie Thomas BEHRMANN, der lange Weg zum Rezess. Das erste Jahrhundert hansischer Versammlungsschriftlichkeit. in: Frühmittelalterliche Studien. Bd. 36, 2002, S. 433-467. Leider hat Thomas Behrmann die Formularveränderung von 1379/80 nicht mehr in seine Betrachtungen mit eingeschlossen.
} 
grund ihrer geographischen Ausgangslage ein Interesse an sehr vielen regionalen Konflikten zeigten. Das Handelsgebiet von Novgorod bis England lag im lübisch-hamburgischen Interessensfeld, und damit waren diese Städte in ständigem Kontakt mit anderen Städtegruppen. Auch die Veränderung der Handelsusancen spielte in diesem Zusammenhang eine Rolle. Als die Kaufleute ihre Waren nicht mehr begleiteten und die Geschäfte vom heimischen Kontor aus regelten, überschauten die wendischen Kaufleute, ebenfalls aufgrund ihrer geographischen Lage, einen weiten ökonomischen Raum und dessen Konflikte. Dieses mündete in einer engeren Zusammenarbeit mit anderen beteiligten Städtegruppen.

Zum zweiten hatte der militärische Konflikt mit Dänemark, der für die Seestädte siegreich ausgegangen war, anderen Gruppen und Städten die Vorteile einer engen Zusammenarbeit und die Macht, die aus einer solchen Zusammenarbeit folgen konnte, vor Augen geführt. So kommt es nicht von ungefähr, dass sich das bis dahin unabhängige Londoner Kontor spätestens Ende der 1370er Jahre an die Seestädte wandte, um sich von ihnen Unterstützung im Kampf gegen Parlament und Krone zu verschaffen - ein Schritt, der für alle Seiten fatale Auswirkungen haben sollte. Die Hinwendung eines Kontors zwang wiederum die Städtegruppe zu noch engerer und vor allem permanenter Zusammenarbeit. Hieraus resultiert die häufigere Anwesenheit von anderen Ratssendboten bei den Versammlungen der Seestädte.

Die Hanse als Institution entwickelte sich also langsam aus den bestehenden Strukturen heraus und verfestigte ihre Strukturen erst am Ende des 14. Jahrhunderts, also nach einer Zeit, die früher gemeinhin als Höhepunkt der Hanse überhaupt gesehen wurde. Sie spiegelt, wie das Kontor in Brügge, die Gruppen wider, die die Gemeinschaft der Hanse gegründet haben. ${ }^{134}$ Sie ist also eine Momentaufnahme der jeweils gegenwärtigen politischen Situation. Zu einem „Hansetag“ erschienen diejenigen, die daraus ökonomische oder politische Vorteile erzielen konnten. Dabei gab es eine Kerngruppe, die aufgrund ihrer geographischen Zentrallage oder ihrer vielfältigen ökonomischen Interessen häufiger vertreten war, wohingegen andere Kaufleute resp. Städte, z. B. Stockholm, Kalmar, Krakau, Mühlhausen in Thüringen oder die holländischen und seeländischen Städte, nur dann teilnahmen, wenn sie ,hansisch" sein wollten. Hanse ergab sich aus der eigenen Interessenlage. ${ }^{140}$

Die Hanse verblieb und agierte sehr erfolgreich in dieser losen Form bis zum Anfang resp. zur Mitte des 15. Jahrhunderts. Am Anfang des neuen

${ }^{139}$ Einleitend Heinz SтоOв, Kaiser Karl IV. und seine Zeit, Graz 1990, S. $140 \mathrm{f}$.

${ }^{140}$ VON BRANDT, Die Hanse (wie Anm. 130), S. 17. 
Jahrhunderts scheint mit der Delegation von Vertretungsaufgaben an Lübeck und neuen inneren Einungsbestrebungen im Jahre 1418 ein nächster Entwicklungsschritt hin zu einer Organisation erreicht zu sein. ${ }^{141}$ Der nächste Schritt erfolgte, als die Hanse durch Verhandlungen mit England gezwungen wurde, sich ihrer Struktur überhaupt erst bewusst zu werden. ${ }^{142}$ Noch zu diesem Zeitpunkt beschrieb sie sich aber selbst mit den folgenden Worten: Die Deutsche Hanse ist keine Gesellschaft, auch keine Gemeinschaft, die durch das weltliche oder kanonische Recht definiert werden kann. Sie hat keine gemeinschaftlichen Angelegenheiten, noch eine gemeinsame Kasse, kein gemeinsames Siegel, keinen gemeinsamen Syndicus, um gemeinschaftliche Verhandlungen zu führen, all das findet sich in der Hanse nicht, ${ }^{143}$ und fährt fort: Die Deutsche Hanse ist keine Gesellschaft, Genossenschaft auch keine Gemeinschaft, aber sie ist eine Gruppe von vielen Marktstädten, Städten und Einrichtungen, die Handel zu See und zu Land zu ihrem Wohle betreiben, in einem Bündnis vereinigt, um Piraten, Räuber und andere Gefahren zu Lande und zu Wasser abzuwehren, so dass ihre Kaufleute und deren Waren nicht geschädigt würden. ${ }^{144}$ Sie selbst definierte sich zudem als einen corpus in etliken vruntscoppen unde vorbintnissen. ${ }^{145}$ Diese Umschreibungen, so unverständlich sie auch für die englischen Unterhändler gewesen sein mussten, geben genau den Zustand wieder, der sich aus der oben gezeigten Entwicklung ergeben hatte. Die Hanse war existent, wenn man sie aus gemeinsamem Interesse benötigte. Sie war keine Organisation, die aus sich heraus existierte. Erst die Konfrontation mit einer am römischen Recht geschulten Staatsmacht leitete weitere Entwicklungsschritte von der Institution zur Organisation ein, weg von dem ad hoc und lose agierenden Verbund, ohne organisatorische Merkmale wie gemeinsames Siegel, Kasse, Syndicus oder Angestellte, hin zu einer Organisation, die teilweise diese Merkmale aufwies

'1 Siehe hierzu ausführlich Volker HENN, Die Hanse: Interessengemeinschaft oder Städtebund? Anmerkungen zu einem neuen Buch, in: HGbll. 102, 1984, S. 119-126, hier S. 122f.

${ }^{142}$ Siehe hierzu grundlegend Ernst PITZ, Bürgereinung und Städteeinung. Studien zur Verfassungsgeschichte der Hansestädte und der Hanse (QDhG 52), Köln 2001, vor allem S. $1-20$.

${ }^{143}$ HUB IX, Nr. 584, § 2, S. 463. Ansam Theutonicam non esse societatem. I...J Non est eciam universitas, quia ad hoc, quod aliqua communitas universitatis nomen mereatur, requiritur tam iure civili quam canonico, quod habeat res communes, communem archam, commune sigillum, communem sindicum et communem actorem, communia negocia gerentem, sed nullum illorum est reperire in Ansa Theutonica.

${ }^{144}$ HUB IX, Nr. 584, $\$ 2$, hier S. 464. Non est Ansa Theutonica societas, collegium vel universitas, sed est multarum civitatum, opidorum et communitatum firma confederacio, ut intercursus negociacionum terra ef mari votivum ac prosperum habaeat successum, ut piratis, latronibus ac aliis predas terra ac mari agentibus resistencia dentur oportuna, ne eorum insidiis negociatores bonis suis er mercibus spolientur.

14. HR II, 3. Nr. 651, \$10, S. 497f. HENN, Die Hanse (wie Anm. 141), S. $119 \mathrm{f}$. 
und damit in sich selbst existent wurde. Es ist daher wichtig, bei der Betrachtung der Hansegeschichte diese verschiedenen institutionellen Phasen voneinander zu unterscheiden.

\section{Die Hanse und ihr Bild in der Geschichtsschreibung}

Bei den vorangegangenen Ausführungen ging es nicht darum, die Entstehung der Hanse einige Jahre vor- oder rückzudatieren. Dieses wäre in der Tat eine vergebliche Liebesmüh. Es ging vielmehr darum, die Grundeinsicht in das Werden der Hanse neu zu justieren. Unser bisheriges Bild dieser Einrichtung ist noch immer viel zu sehr von den Staatsbegriffen (und der Staatsbegrifflichkeit) des 19. Jahrhunderts geprägt. Die Hanse wurde weder gegründet, noch hatte sie einen eigenen Machtwillen und schon gar keinen Aufstieg, Fall oder Niedergang. ${ }^{146}$ Die Hanse hatte vor allem keinen „Höhepunkt" um 1370, ${ }^{147}$ zu diesem Zeitpunkt war sie höchstens als abstrakter Gedanke, aber keinesfalls als Institution und schon gar nicht als Organisation, existent. Die Hanse entwickelte sich vielmehr in Anpassung an bestimmte Situationen, wurde von ihren Mitgliedern institutionalisiert und dann organisiert, als sie sich einen Vorteil daraus erhoffen konnten, und entwickelte sich in dieser offenen Form weiter. Einige Städte, so die wendischen, waren daran interessiert, die lokalen Konflikte, die sie selbst und ihren Handel berührten, vor dem Forum behandeln zu lassen, das ihnen am nächsten lag: dem Hansetag. Andere Regionen konnten dieses ebenfalls als sinnvoll erachten - so beschickten sie den Tag, oder aber sie zeigten kein Interesse an einer Einmischung dieser Städte, und so beschickten sie den Tag nicht. Die Hanse erhielt somit so viel Macht, wie ihre Mitglieder ihr geben wollten und für notwendig erachteten. Dass die wendischen Städte dabei begonnen hatten, neben politischen und handelspolitischen Fragen auch ökonomische Sachverhalte zu regeln, die die Transaktionskosten wesentlich minderten, machte die Hanse für viele Gruppen - über das Politische hinaus - interessant und erklärt, warum dieser Städtebund eine längere Lebensdauer besaß, als die üblichen regionalen Bünde. Darüber hinaus war die Teilhabe an der Hanse bis ins 16. Jahrhundert hinein nicht nur freiwillig, sondern auch kostenlos. Die Hanse war daher ohne Probleme nutzbar, wenn man sie denn brauchte.

\footnotetext{
${ }^{1}+6$ Ich danke Frau Angela Huang, Kopenhagen, für die langen und anregenden Diskussionen zu diesem Thema. Gerade der Hinweis auf die Probleme mit dem Entwicklungsbegriff der Hanse stammen von ihr und wurden permanent weiterentwickelt.

${ }^{147}$ Diese Bewertung beruht vor allem auf den Forschungen Rörigs. Siehe hierzu Philippe DOLlinger. Die Bedeutung des Stralsunder Friedens in der Geschichte der Hanse, in: HGbll, 88, 1970, S. 148-162, hier S. 148f. - Siehe zur Kritik an diesem Gedanken schon vON BrandT, Die Hanse (wie Anm. 130), S. 16 f.
} 
Die Hanse war kein Staat und sie hatte keine Staatsstruktur, dieses ist in der modernen Hanseforschung ein Gemeinplatz. Die Entstehung und die funktionellen Grundlagen dieser Institution und späteren Organisation zeigen aber auch, warum dieses so ist.

Betrachtet man die Entstehung der Hanse unter diesen Gesichtspunkten, so ist auch das Verhältnis der Hanse zu den binnenländischen Städten neu zu bewerten. Die ältere Hanseforschung, verleitet durch die häufige Verwendung des Begriffes der ,civitates maritimae“ in den zu Hanserezessen deklarierten Dokumenten, hat sich nicht nur allzu sehr auf die Seestädte konzentriert, sondern sie konnte auch gleichzeitig konstatieren, dass die Binnenstädte bei den wichtigen Aktionen der angeblichen „Hanse“ gar nicht teilnahmen oder sich „erstaunlich passiv" verhielten. Das wiederum hat zu dem Problem geführt, dass man nicht erklären konnte, warum diese Institution, der mehr Binnen- als Seestädte angehörten, überhaupt Binnenstädte als Mitglieder in ihren Reihen zählte. Als Resultat hat die seelastige Hanseforschung die Binnenstädte zumeist ignoriert oder nur am Rande erwähnt, ohne deren Bedeutung erklären zu können.

Sieht man das Verhältnis nun unter einem anderen Blickwinkel so fallen zwei Dinge ins Auge: Zum einen verhielten sich die Binnenstädte bis zum Ende des 14. Jahrhunderts nicht ,passiv“, sondern sie nahmen z. B. am Krieg gegen Dänemark gar nicht teil, da sie nicht Teil des Bündnisses waren und dieses auch außerhalb ihres Interessefeldes lag. Sie konnten sich passiv-solidarisch verhalten, z. B. durch Zahlung von Pfundzoll durch ihre Kaufleute, aber der Krieg gegen Dänemark war nicht ihrer und lag nicht in ihrem näheren oder weiteren Interessenfeld. ${ }^{148} \mathrm{Zu}$ anderen Zeitpunkten und zu anderen Gelegenheiten hatten sie auch schon vorher mit den Seestädten zusammengearbeitet, aber zur Mitte des 14. Jahrhunderts lag ihr Interessenschwerpunkt woanders. Zum zweiten stießen die Binnenstädte erst zur Hanse und beteiligten sich erst an den Aktivitäten der Hanse, als die Hanse institutionelle Regeln schuf, die den Handel und die Produktion dieser Städte direkt oder indirekt beeinflussten. Regeln für den Verkauf von hansischem Tuch auf dem Markt von London hatten Einfluss auf die Produktion dieser Tuche in Westfalen oder Sachsen. ${ }^{149}$ Hier also nahm die Hanse eine wichtige Rolle als Vermittler von Informationen ein, auch für Städte, deren Kaufleute nun nicht mehr direkt auf die ausländischen Märkte reisten, deren heimischer Markt aber von den „großen“ ökonomischen Entwicklungen abhängig war. Insofern ist es nicht verwunderlich, dass eine ganze Reihe binnenländischer Städte sich der Hanse an-

\footnotetext{
${ }^{148}$ Siehe hierzu schon HENN, ,... de alle tyd" (wie Anm. 109), S. 40 f.

${ }^{149}$ Siehe hierzu in Zukunft maßgeblich Angela HUANG. Die Textilien des Hanseraums (wie Anm. 57).
} 
schlossen, und das, obwohl sie nie ihre Ratssendboten auf den Hansetag schickten und sie kein offensichtliches Interesse an den politischen Entwicklungen in England oder Dänemark zeigten.

Auch diese Städte waren selbstverständlich in die regionalen Städtebünde eingebunden. Das war der naheliegende Fixpunkt ihrer politischen Aktivitäten. ${ }^{150}$ Es ist daher logisch, dass sich die Städte zu Vororten für die Vertretung der kleinen Städte in der Hanse entwickelten, die auch in den Städtebünden die führende Rolle einnahmen. Auch hier spiegelt die Hanse die regionalen Strukturen (und deren Veränderung im Laufe der Zeit) wider. In diesem Zusammenhang benötigt die Hanseforschung eine tiefere Zusammenarbeit mit der Regionalgeschichte der einzelnen Gebiete, um das Zusammenspiel von Region, regionalem Bund, Produktion und Konsum sowie Hanse besser beleuchten zu können.

Die Hanse war die Folge einer langen Reihe verschiedener Entwicklungen. Im Laufe ihrer Geschichte von 1379 bis ins 17. Jahrhundert wurde sie laufend an die Bedingungen der Zeit angepasst, bis hin zu ihrer Abwicklung, als man sie nicht mehr benötigte. Die Institutionalisierung der Hansetage ab 1379/80 und die Schaffung einer Organisation im 15. Jahrhundert führten natürlich zu einer Verstetigung der Verhältnisse und damit auch zu einem institutionellen Konservatismus. Da aber die Hanse nur so viel Macht besaß, wie ihre Glieder ihr in jeder einzelnen Situation geben wollten, konnte sie nur begrenzt eine eigene Machtstruktur entwickeln. Das beständige Suchen nach einem tragbaren Kompromiss, das die Hansetage des 15. und 16. Jahrhunderts auszeichnet, ist die logische Folge ihres Entstehens und ihres Wirkungsmechanismus. Insofern sollte man nicht von der Hanse als einer eigenständig handelnden Organisation sprechen, die ein eigenes Leben (und eine eigene Macht), sowie eine organische Entwicklung aufwies. Die Hanse konnte von ihren Mitgliedern genutzt werden, um ihre Interessen durchzusetzen, aber sie besaß, zumindest im ausgehenden 14. und beginnenden 15. Jahrhundert, niemals ein eigenständiges Leben oder Wirken.

${ }^{150}$ Siehe hierzu u. a. DISTler, Städtebünde (wie Anm. 13), S. $56 \mathrm{f}$. 